\title{
Truncated EM numerical method for generalised Ait-Sahalia-type interest rate model with delay
}

\author{
Coffie Emmanuel* and Xuerong Mao \\ Department of Mathematics and Statistics, \\ University of Strathclyde, Glasgow G1 1XH, U.K.
}

\begin{abstract}
The original Ait-Sahalia model of the spot interest rate proposed by Ait-Sahalia assumes constant volatility. As supported by several empirical studies, volatility is never constant in most financial markets. From application viewpoint, it is important we generalise the Ait-Sahalia model to incorporate volatility as a function of delay in the spot rate. In this paper, we study analytical properties for the true solution of this model and construct several new techniques of the truncated Euler-Maruyama (EM) method to study properties of the numerical solutions under the local Lipschitz condition plus Khasminskii-type condition. Finally, we justify that the truncated EM approximate solution can be used within a Monte Carlo scheme for numerical valuations of some financial instruments such as options and bonds.
\end{abstract}

Key words: Stochastic interest rate model, Delay volatility, Truncated EM scheme, Strong convergence, Monte Carlo scheme.

\section{Introduction}

Stochastic modelling of interest rates plays significant roles in calibration and valuation of financial instruments. Many well-known stochastic models have been proposed to model dynamics of interest rates, for example Black-Scholes (1973) [1], Merton (1973) [2], Vasicek (1977) [3], Dothan (1978) 4], Brennan and Schwartz (1980) [5] and Cox, Ingersoll and Ross(CIR) (1985) [7]. These models were generalised by Lewis (2000) [8] as a non-linear mean-reverting-theta process

$$
d x(t)=\alpha(\mu-x(t)) d t+\sigma x(t)^{\theta} d B(t)
$$

$x(0)=x_{0}$, for any $t>0$, where $\alpha, \mu$ and $\sigma$ are constants, $\theta \geq 1 / 2$ and $B$ is a scalar Brownian motion. This is widely used to model dynamics of interest rates, volatility and other financial quantities. Mao [11] studied analytical properties and strong convergence theory of the numerical solutions of SDE (1) when $\theta \in[1 / 2,1]$. Higham and Mao [9] examined the strong convergence of Monte Carlo simulations of SDE (1). Wu [10] established analytical properties of SDE (1) and convergence of EM approximate solutions in probability when $\theta>1$.

Ait-Sahalia [12] conducted empirical studies to investigate several continuous-time interest rate models. He tested parametric models by comparing their implied parametric density to the same

${ }^{*}$ Corresponding author, Email: emmanuel.coffie@strath.ac.uk 
density estimated nonparametrically. He discovered all existing univariate linear drift models could not explain well the dynamics of Eurodollar interest rates. As a result, he proposed a new class of strongly non-linear SDE described by

$$
d x(t)=\left(\alpha_{-1} x(t)^{-1}-\alpha_{0}+\alpha_{1} x(t)-\alpha_{2} x(t)^{2}\right) d t+\sigma x(t)^{\theta} d B(t)
$$

$x(0)=x_{0}$, for any $t>0$, where $\alpha_{-1}, \alpha_{0}, \alpha_{1}, \alpha_{2}$ are positive constants and $\theta>1$, for modelling interest rate dynamics. In his seminal paper, Ait-Sahalia used Feller test to show conditions under which almost surely the solution of SDE (2) will not explode in finite time to infinity. Cheng [13] studied analytical properties including nonnegativity of solution and boundedness of moments of SDE (2) and established convergence of EM approximate solution to the true solution in probability. Szpruch et al. in [14] generalised SDE (2) to

$$
d x(t)=\left(\alpha_{-1} x(t)^{-1}-\alpha_{0}+\alpha_{1} x(t)-\alpha_{2} x(t)^{\rho}\right) d t+\sigma x(t)^{\theta} d B(t), \quad x(0)=x_{0},
$$

for any $t>0$ and established analytical properties and strong convergence of backward and forwardbackward EM approximate solutions to the exact solution of this type of model when $\rho>1$. Dung (2016) in [15] derived explicit estimates for tail probabilities for solutions to SDE (3). Interestingly, all the aforementioned interest rate models share a common characteristic, i.e. volatility is assumed constant. However, several empirical studies proved that volatility is not constant in most financial markets and any good financial model should possess important characteristic of reproducing volatility 'smiles' and 'skews' evident in option markets (e.g., see [17, 20] for detailed accounts).

There are several extensive literature where stochastic models with inherent features of past dependency are used to describe volatility 'smiles' and 'skews' adequately. For instance, Kind et al. justified in [19] that the instantaneous volatility is modelled in terms of the sample variance of the log-prices over a past interval of fixed length. Mao and Sabanis [20] also extended the geometric Brownian motion $(\mathrm{GBM})$ to a delay geometric Brownian motion (DGBM) described by a stochastic delay differential equation (SDDE), where the volatility is modelled as a function of delay in asset price and justified it as a rich alternative for modelling financial instruments in a complete market.

In this light, it is natural to introduce delayed volatility function into the generalised strongly nonlinear Ait-Sahalia model described by the stochastic delay differential equation (SDDE )

$$
\begin{cases}d x(t)=\left(\alpha_{-1} x(t)^{-1}-\alpha_{0}+\alpha_{1} x(t)-\alpha_{2} x(t)^{\rho}\right) d t+V(x(t-\tau)) x(t)^{\theta} d B(t), & t \geq 0, \\ x(t)=\xi(t), & t \in[-\tau, 0],\end{cases}
$$

for evolution of interest rates. Here $\alpha_{-1}, \alpha_{0}, \alpha_{1}, \alpha_{2}$ are positive constants, $\rho, \theta>1$ are parameters, $B(\cdot)$ is a scalar Brownian motion and $V(\cdot)$ is a volatility function which depends on $x(t-\tau)$, where $\tau>0$ and $x(t-\tau)$ denotes delay in $x(t)$. The delayed volatility function $V(x(t-\tau))$ is past-level-dependent in this case and hence may capture dynamics of volatility 'smiles' and 'skews' adequately.

The solution to SDDE (4) obviously cannot be found by closed-form formulas. It is also obvious SDDE (4) has superlinear coefficient terms. This is further complicated by $\alpha_{-1} x(t)^{-1}$ in the drift term, which may explode to infinity in finite time at the origin, and $V(x(t-\tau))$ in the diffusion term. Hence we cannot employ standadrd EM scheme to numerically approximate SDDE (4) since this scheme diverges in strong mean-square sense at finite point for SDEs with superlinear coefficients (see [16]). The truncated EM scheme was developed recently in 21] for numerical approximation of SDEs with superlinear coefficients. However, the truncated EM scheme may fail to cope with SDDE (4).

In this work, we are concerned with constructing several new techniques of the truncated EM scheme to numerically study SDDE (4) and establish strong convergence theory in finite time under 
the local Lipschitz condition plus Khasminskii-type condition. In particular, we aim to establish $L^{q}$ strong convergence theory in finite time, where $L^{q} \supset L^{p}, q \in[2, p)$ and $p$ is a parameter in connection with the Khasminskii-type condition.

The rest of the paper is organised as follows: In section 2, we will verify the existence and uniqueness for the solution to SDDE (4) and show that the solution will never become negative. We will also study analytical properties such as boundedness of pth moment of the true solution in section 2 . In section 3, we will construct the truncated EM scheme for SDDE (4). We will explore numerical properties and investigate a finite time strong convergence of this scheme in section 4 . In section 5, we will apply the strong convergence result within a Monte Carlo setting to value some financial instruments. Finally, we will perform some numerical examples to support the established results in section 6 .

\section{Properties of the generalised Ait-Sahalia model with delay}

\subsection{Mathematical preliminaries}

Throughout this paper unless specified otherwise, we employ the following notation. Let $\{\Omega, \mathcal{F}, \mathbb{P}\}$ be a complete probability space with filtration $\left\{\mathcal{F}_{t}\right\}_{t \geq 0}$ satisfying the usual conditions (i.e., it is increasing and right continuous while $\mathcal{F}_{0}$ contains all $\mathbb{P}$ null sets), and let $\mathbb{E}$ denote the expectation corresponding to $\mathbb{P}$. Let $B(t), t \geq 0$, be a scalar Brownian motion defined on the above probability space. If $x, y$ are real numbers, then $x \vee y$ denotes the maximum of $x$ and $y$, and $x \wedge y$ denotes the minimum of $x$ and $y$. Let $\mathbb{R}=(-\infty, \infty)$ and $\mathbb{R}_{+}=(0, \infty)$. For $\tau>0$, let $C\left([-\tau, 0] ; \mathbb{R}_{+}\right)$denote the space of all continuous functions $\xi:[-\tau, 0] \rightarrow \mathbb{R}_{+}$with the norm $\|\xi\|=\sup _{-\tau \leq t \leq 0} \xi(t)$. For an empty set $\emptyset$, we set inf $\emptyset=\infty$. For a set $A$, we denote its indication function by $1_{A}$. Consider the following nonlinear dynamics

$$
d x(t)=f(x(t)) d t+V(x(t-\tau)) g(x(t)) d B(t),
$$

$x(t)=\xi(t)$, on $t \in[-\tau, \infty)$, as equation of SDDE (4) such that $f(x)=\alpha_{-1} x^{-1}-\alpha_{0}+\alpha_{1} x-\alpha_{2} x^{\rho}$ and $g(x)=x^{\theta}, \forall x \in \mathbb{R}_{+}$, with $V(\cdot)$ defined in $C\left(\mathbb{R}_{+} ; \mathbb{R}_{+}\right)$. Let $C^{2,1}\left(\mathbb{R} \times \mathbb{R}_{+} ; \mathbb{R}\right)$ be the family of all real-valued functions $H(x, t)$ defined on $\mathbb{R} \times \mathbb{R}_{+}$such that $H(x, t)$ is twice continuously differentiable in $x$ and once in $t$. Given $H \in C^{2,1}\left(\mathbb{R} \times \mathbb{R}_{+} ; \mathbb{R}\right)$, define an operator $L H: \mathbb{R} \times \mathbb{R} \times \mathbb{R}_{+} \rightarrow \mathbb{R}$ by

$$
L H(x, y, t)=H_{t}(x, t)+H_{x}(x, t) f(x)+\frac{1}{2} H_{x x}(x, t) V(y)^{2} g(x)^{2},
$$

called the diffusion operator of the dynamics (5) associated with the $C^{2,1}$-function $H$, where

$$
H_{t}(x, t)=\frac{\partial H(x, t)}{\partial t}, H_{x}(x, t)=\frac{\partial H(x, t)}{\partial x} \text { and } H_{x x}(x, t)=\frac{\partial^{2} H(x, t)}{\partial x^{2}} .
$$

With the diffusion operator, the Itô formula can be written as

$$
d H(x(t), t)=L H(x(t), x(t-\tau), t) d t+H_{x}(x(t), t) V(x(t-\tau)) g(x(t)) d B(t) \quad \text { a.s. }
$$

See, for instance, [1] for further details.

\section{$2.2 \quad$ Existence of nonnegative solution}

We have already observed the $f$ and $g$ coefficient terms of SDDE (5) are non-globally Lipschitz continuous. Naturally, for SDDE (5) to have a unique global solution for any given initial data, both drift 
and diffusion terms are required to satisfy local Lipschitz condition plus superlinear growth condition (e.g., see [14] for more details). Essentially, this requires to assume that the volatility function $V(\cdot)$ is locally Lipschitz continuous and bounded. The following theorem illustrates that the SDDE (5) admits a unique positive global solution. Moreover, since the SDDE (5) describes interest rate dynamics in the financial market, it is important the solution $x(t)$ should always be positive. The following conditions are however sufficient to establish a pathwise-unique positive global solution $x(t)$ to SDDE (5).

Assumption 2.1. (Boundedness of volatility function). The volatility function $V: \mathbb{R}_{+} \rightarrow \mathbb{R}_{+}$of SDDE (5) is Borel-measurable and bounded by a positive constant $\lambda$, that is

$$
V(y) \leq \lambda, \quad \forall y \in \mathbb{R}_{+}
$$

See, for instance, Mao and Sabanis [20] for detailed coverage of the above assumption. In addition to Assumption 2.1, we also require the following assumption on the parameter values to help control the potential growth likely to emerge from the diffusion term.

Assumption 2.2. (Condition on parameter values). The parameters of the SDDE (5) satisfy

$$
1+\rho>2 \theta \text {. }
$$

Theorem 2.3. Let Assumptions 2.1 and 2.2 hold. Then for any given initial data

$$
\{x(t):-\tau \leq t \leq 0\}=\xi(t) \in C([-\tau, 0]): \mathbb{R}),
$$

there exists a unique positive global solution $x(t)$ to $\mathrm{SDDE}(5)$ on $t \geq 0$. This solution can be computed by the following step by step procedure: for $k=0,1,2, \ldots$ and $t \in[k \tau,(k+1) \tau]$,

$$
x(t)=x(k \tau)+\int_{k \tau}^{t} f(x(s)) d s+\int_{k \tau}^{t} V(x(s-\tau)) g(x(s)) d B(s) .
$$

Moreover, for any $T>0$,

$$
\lim _{n \rightarrow \infty} \mathbb{P}\left(\tau_{n} \leq T\right)=0,
$$

where $\tau_{n}=\inf \{t \geq 0: x(t) \notin(1 / n, n)\}$ for every sufficiently large integer $n$.

We employ an inductive argument to establish this proof.

Proof. For $t \in[0, \tau]$, the SDDE (5) becomes the following SDE

$$
d x(t)=f(x(t)) d t+V(\xi(t-\tau)) g(x(t)) d B(t),
$$

with initial value $x(0)=\xi(0)$ and has a well-known unique positive global solution

$$
x(t)=\xi(0)+\int_{0}^{t} f(x(s)) d s+\int_{0}^{t} V(\xi(s-\tau)) g(x(s)) d B(s) .
$$

The solution $x(t)$ to SDE (12) on $t \geq 0$ has been however established in various literature to satisfy (11) (see, e.g., [12, 14] for more details). This implies (10) holds for $k=0$. As $x(t)$ is now known on $t \in[0, \tau]$, we may repeat this procedure over the interval $t \in[\tau, 2 \tau]$ to obtain the SDE

$$
d x(t)=f(x(t)) d t+V(x(t-\tau)) g(x(t)) d B(t) .
$$


This SDE has a unique positive global solution

$$
x(t)=\xi(0)+\int_{0}^{t} f(x(s)) d s+\int_{0}^{t} V(x(s-\tau)) g(x(s)) d B(s) .
$$

Clearly the solution $x(t)$ is a continuous stochastic process on $t \in[0, \tau]$ and so both integrals are well defined. Hence the (10) holds for $k=1$. Given that the solution $x(t)$ to SDE (12) on $t \geq 0$ satisfies (11) implies it also satisfies (11) for SDE (13). Repeating this procedure for all $k \geq 0$, we obtain a unique positive global solution to SDDE (5) which satisfies (11).

\subsection{Moment bound of the true solution}

Moment bounds are essential for valuation and pricing of financial quantities. The following lemmas give boundedness properties for the solution to SDDE (5).

Lemma 2.4. Let Assumptions 2.1 and 2.2 hold. Then for any $p \geq 2$, the solution $x(t)$ to SDDE (5) is upper bounded, i.e.,

$$
\sup _{0 \leq t<\infty}\left(\mathbb{E}|x(t)|^{p}\right) \leq C_{1}
$$

for any $t \geq 0$ and

$$
\sup _{0 \leq t<\infty}\left(\mathbb{E}\left|\frac{1}{x(t)}\right|^{p}\right) \leq C_{2}
$$

where $C_{1}$ and $C_{2}$ are constants.

Proof. For every sufficiently large integer $n$, define the stopping time by

$$
\tau_{n}=\inf \{t \geq 0: x(t) \notin(1 / n, n)\} .
$$

Apply the diffusion operator to $H(x, t)=e^{t} x^{p}$ to get

$$
\begin{aligned}
L H(x, y, t) & =e^{t} x^{p}+p e^{t} x^{p-1} f(x)+\frac{1}{2} p(p-1) e^{t} x^{p-2}(V(y) g(x))^{2} \\
& =e^{t} x^{p}+p e^{t} x^{p-1}\left(\alpha_{-1} x^{-1}-\alpha_{0}+\alpha_{1} x-\alpha_{2} x^{\rho}\right)+\frac{1}{2} p(p-1) e^{t} x^{p-2} V^{2}(y) x^{2 \theta} \\
& \leq e^{t}\left[x^{p}+p x^{p-2}\left(\alpha_{-1}-\alpha_{0} x+\alpha_{1} x^{2}-\alpha_{2} x^{\rho+1}+\frac{(p-1)}{2} \lambda^{2} x^{2 \theta}\right)\right]
\end{aligned}
$$

By Assumption 2.2, there exists a constant $K$ such that

$$
L H(x, y, t) \leq e^{t} K
$$

By the Itô formula,

$$
\begin{aligned}
\mathbb{E}\left[e^{t \wedge \tau_{n}}\left|x\left(t \wedge \tau_{n}\right)\right|^{p}\right] & \leq|\xi(0)|^{p}+\mathbb{E} \int_{0}^{t \wedge \tau_{n}} K e^{s} d s \\
& \leq|\xi(0)|^{p}+K e^{t} .
\end{aligned}
$$

Applying the Fatou lemma and letting $n \rightarrow \infty$ gives

$$
\mathbb{E}|x(t)|^{p} \leq \frac{|\xi(0)|^{p}}{e^{t}}+K<\infty
$$

and hence

$$
\sup _{0 \leq t<\infty}\left(\mathbb{E}|x(t)|^{p}\right) \leq C_{1}
$$


We can show (15) the same way by applying the Itô formula to $H(x, t)=e^{t} x^{-p}$.

Lemma 2.5. Let Assumptions 2.1 and 2.2 hold. Then for any $p \geq 2$, the solution $x(t)$ to SDDE (5) satisfies

$$
\mathbb{E}\left(\sup _{0 \leq t \leq T}|x(t)|^{p}\right) \leq C_{3}
$$

on $t \geq 0$, where $C_{3}$ is a constant.

Proof. Define a function $H \in C^{2}\left(\mathbb{R}_{+}, \mathbb{R}_{+}\right)$by

$$
H(x)=x^{p}
$$

By the Itô formula,

$$
\begin{aligned}
d H(x(t)) & =p x^{p-1} d x(t)+\frac{1}{2} p(p-1) x^{p-2}(d x(t))^{2} \\
& =p x^{p-1}\left(\alpha_{-1} x(t)^{-1}-\alpha_{0}+\alpha_{1} x(t)-\alpha_{2} x(t)^{\rho}+\frac{1}{2} p(p-1) x(t)^{2(\theta-1)+p} V(y)^{2}\right) d t \\
& +p x(t)^{p+\theta-1} V(y) d B(t) \\
& \leq\left[p x^{p-2}\left(\alpha_{-1}-\alpha_{0} x(t)+\alpha_{1} x(t)^{2}-\alpha_{2} x(t)^{\rho+1}+\frac{(p-1)}{2} \lambda^{2} x(t)^{2 \theta}\right)\right] d t+\lambda p x(t)^{p+\theta-1} d B(t) .
\end{aligned}
$$

So

$$
\begin{aligned}
\mathbb{E}\left(\sup _{0 \leq t \leq T}|x(t)|^{p}\right) & \leq|\xi(0)|^{P}+\mathbb{E} \int_{0}^{T}\left[p x ^ { p - 2 } \left(\alpha_{-1}-\alpha_{0} x(t)+\alpha_{1} x(t)^{2}-\alpha_{2} x(t)^{\rho+1}\right.\right. \\
& \left.\left.+\frac{(p-1)}{2} \lambda^{2} x(t)^{2 \theta}\right)\right] d t+\mathbb{E}\left[\sup _{0 \leq t \leq T} \int_{0}^{t} \lambda p x(s)^{p+\theta-1} d B(s)\right] .
\end{aligned}
$$

By Assumption 2.2, there exists a constant $K$ such that

$$
\mathbb{E}\left(\sup _{0 \leq t \leq T}|x(t)|^{p}\right) \leq|\xi(0)|^{P}+K T+\mathbb{E}\left[\sup _{0 \leq t \leq T} \int_{0}^{t} \lambda p x(s)^{p+\theta-1} d B(s)\right] .
$$

By the Hölder and Burkholder-Davis Gundy inequalities we then obtain,

$$
\mathbb{E}\left(\sup _{0 \leq t \leq T}|x(t)|^{p}\right) \leq|\xi(0)|^{P}+K T+C\left(\int_{0}^{T} \mathbb{E} x(s)^{2(p+\theta-1)} d s\right)^{1 / 2},
$$

where $C$ is a constant which may vary from line to line. Hence

$$
\mathbb{E}\left(\sup _{0 \leq t \leq T}|x(t)|^{p}\right) \leq C_{3}
$$




\section{The truncated EM method}

As we have already noted, the truncated EM method for SDEs under local Lipschitz condition plus Khasminskii-type condition was developed in [21]. This numerical method was further developed in [22] to study SDDEs under local Lipschitz condition plus generalised Khasminskii-type condition. Hence, in order to study SDDE (5) using the truncated EM techniques, we require the following conditions on the coefficient terms.

Assumption 3.1. (Local Lipschitz continuity of $V$ ). For any $R>0$, there exists a positive constant $L_{R}$ such that volatility function $V(\cdot)$ in the diffusion term of SDDE (5) satisfies

$$
|V(y)-V(\bar{y})| \leq L_{R}|y-\bar{y}|
$$

for all $y, \bar{y} \in\left[\frac{1}{\mathbb{R}}, \mathbb{R}\right]$.

Lemma 3.2. (Local Lipschitz continuity of $f$ and $g$ ). For any $R>0$, there exists a positive constant $K_{R}>0$ such that the $f$ and $g$ coefficient terms of SDDE (5) satisfy

$$
|f(x)-f(\bar{x})| \vee|g(x)-g(\bar{x})| \leq K_{R}|x-\bar{x}|
$$

for all $x, \bar{x} \in\left[\frac{1}{\mathbb{R}}, \mathbb{R}\right]$.

Lemma 3.3. (Khasminskii-type condition). Let Assumptions 2.1 and 2.2 hold. For any $p \geq 2$, there exists $K_{1}=K(p)>0$ such that the coefficients of SDDE (5) satisfy

$$
x f(x)+\frac{p-1}{2}|V(y) g(x)|^{2} \leq K_{1}\left(1+|x|^{2}\right)
$$

for all $x, y \in \mathbb{R}_{+}$.

Proof. By Assumption 2.1, $\forall x, y>0$, we have that

$$
\begin{aligned}
x f(x)+\frac{p-1}{2}|V(y) g(x)|^{2} & =x\left(\alpha_{-1} x^{-1}-\alpha_{0}+\alpha_{1} x-\alpha_{2} x^{\rho}\right)+\frac{p-1}{2}\left|V(y) x^{\theta}\right|^{2} \\
& \leq \alpha_{-1}-\alpha_{0} x+\alpha_{1} x^{2}-\alpha_{2} x^{\rho+1}+\frac{p-1}{2} \lambda^{2} x^{2 \theta} .
\end{aligned}
$$

By Assumption 2.2,

$$
\begin{aligned}
x f(x)+\frac{p-1}{2}|V(y) g(x)|^{2} & \leq \alpha_{-1}-\alpha_{0} x+\alpha_{1} x^{2}+K(p) \\
& \leq \alpha_{-1}+\alpha_{1} x^{2}+K(p) \\
& \leq K_{1}\left(1+|x|^{2}\right)
\end{aligned}
$$

where $K(p) \geq-\alpha_{2} x^{\rho+1}+\frac{p-1}{2} \lambda^{2} x^{2 \theta}$ and $K_{1}=\left[\left(\alpha_{-1}+K(p)\right) \vee \alpha_{1}\right]$.

\subsection{The truncated EM approximation}

Before we proceed to construct the truncated EM scheme, let us extend the domain of the volatility function $V(\cdot)$ from $\mathbb{R}_{+}$to $\mathbb{R}$ by setting the volatility function $V(x)=V(0)$ for $x<0$. It is worth to note that the solution for the SDDE (5) is already known to always be positive, so this extension 
does not in any way influence the solution. The local Lipschitz condition in Assumption 3.1 and the boundedness condition on $V(\cdot)$ in (8) are also well preserved.

To define the truncated EM numerical solutions for the SDDE (5), we first choose a strictly increasing continuous function $\mu: \mathbb{R}_{+} \rightarrow \mathbb{R}_{+}$such that $\mu(r) \rightarrow \infty$ as $r \rightarrow \infty$ and

$$
\sup _{1 / r \leq x \leq r}(|f(x)| \vee g(x)) \leq \mu(r), \quad \forall r>1
$$

Denote by $\mu^{-1}$ the inverse function of $\mu$. We define a strictly decreasing function $h:(0,1) \rightarrow \mathbb{R}_{+}$such that

$$
\lim _{\Delta \rightarrow 0} h(\Delta)=\infty \text { and } \Delta^{1 / 4} h(\Delta) \leq 1, \quad \forall \Delta \in(0,1]
$$

Find $\Delta^{*} \in(0,1)$ such that $\mu^{-1}\left(h\left(\Delta^{*}\right)\right)>1$ and $f(x)>0$ for $0<x<\Delta^{*}$. For a given step size $\Delta \in\left(0, \Delta^{*}\right)$, let us define the truncated functions

$$
f_{\Delta}(x)=f\left(1 / \mu^{-1}(h(\Delta)) \vee\left(x \wedge \mu^{-1}(h(\Delta))\right)\right), \quad \forall x \in \mathbb{R}
$$

and

$$
g_{\Delta}(x)= \begin{cases}g\left(x \wedge \mu^{-1}(h(\Delta))\right), & \text { if } x \geq 0 \\ 0, & \text { if } x<0 .\end{cases}
$$

That is, for $x<1 / \mu^{-1}(h(\Delta))$, we have $f_{\Delta}(x)=f\left(1 / \mu^{-1}(h(\Delta))\right)$ and $g_{\Delta}(x)=0$. For $x>\mu^{-1}(h(\Delta))$, we have $f_{\Delta}(x)=f\left(\mu^{-1}(h(\Delta))\right)$ and $g_{\Delta}(x)=g\left(\mu^{-1}(h(\Delta))\right)$. Moreover, for $x \in\left[1 / \mu^{-1}(h(\Delta)), \mu^{-1}(h(\Delta))\right]$, we have $f_{\Delta}(x)=f(x)$ and $g_{\Delta}(x)=g(x)$ with

$$
\begin{aligned}
\left|f_{\Delta}(x)\right| & =|f(x)| \leq \underset{1 / \mu^{-1}(h(\Delta)) \leq z \leq \mu^{-1}(h(\Delta))}{\max }|f(z)| \\
& \leq \mu\left(\mu^{-1}(h(\Delta))\right) \\
& =h(\Delta)
\end{aligned}
$$

and

$$
g_{\Delta}(x) \leq \mu\left(\mu^{-1}(h(\Delta))\right)=h(\Delta)
$$

It is easy to see that

$$
\left|f_{\Delta}(x)\right| \vee g_{\Delta}(x) \leq h(\Delta), \quad \forall x \in \mathbb{R}
$$

Obviously, both truncated functions $f_{\Delta}$ and $g_{\Delta}$ are bounded although both $f$ and $g$ may not. The following lemma illustrates $f_{\Delta}$ and $g_{\Delta}$ preserve the Khasminskii-type condition in (23) very well.

Lemma 3.4. Let Assumption 2.1 and 2.2 hold. Then, for all $\Delta \in\left(0, \Delta^{*}\right)$ and $p \geq 2$, the truncated functions satisfy

$$
x f_{\Delta}(x)+\frac{p-1}{2}\left|V(y) g_{\Delta}(x)\right|^{2} \leq \bar{K}\left(1+|x|^{2}\right)
$$

$\forall x, y \in \mathbb{R}$, where $\bar{K}$ is a positive constant independent of $\Delta$.

Proof. Fix any $\Delta \in\left(0, \Delta^{*}\right)$. For $x, y \in \mathbb{R}$ with $x \in\left[1 / \mu^{-1}(h(\Delta)), \mu^{-1}(h(\Delta))\right]$, by (27), we have

$$
x f_{\Delta}(x)+\frac{p-1}{2}\left|V(y) g_{\Delta}(x)\right|^{2}=x f(x)+\frac{p-1}{2}|V(y) g(x)|^{2} \leq K_{1}\left(1+|x|^{2}\right)
$$


as the required assertion. For $x \in \mathbb{R}$ with $x \in\left(0,1 / \mu^{-1}(h(\Delta))\right)$, we have

$$
0<x \mu^{-1}(h(\Delta))<1 \text {. }
$$

So

$$
\begin{aligned}
x f_{\Delta}(x) & =x f\left(1 / \mu^{-1}(h(\Delta))\right) \\
& =x \mu^{-1}(h(\Delta)) \frac{1}{\mu^{-1}(h(\Delta))} f\left(1 / \mu^{-1}(h(\Delta))\right) \\
& \leq x \mu^{-1}(h(\Delta))\left(1+\left[1 / \mu^{-1}(h(\Delta))^{2}\right]\right) \\
& \leq K_{1}(1+1)=2 K_{1} .
\end{aligned}
$$

Therefore, we have

$$
\begin{aligned}
x f_{\Delta}(x)+\frac{p-1}{2}\left|V(y) g_{\Delta}(x)\right|^{2} & \leq 2 K_{1}+\frac{p-1}{2}\left|V(y) g_{\Delta}(x)\right|^{2} \\
& \leq 2 K_{1}+\frac{p-1}{2} \lambda^{2} g_{\Delta}(x)^{2} \\
& \leq 2 K_{1}+\frac{p-1}{2} \lambda^{2} \\
& \leq K_{2}\left(1+|x|^{2}\right),
\end{aligned}
$$

where $K_{2}=2 K_{1}+\frac{p-1}{2} \lambda^{2}$. But for $x, y \in \mathbb{R}$ with $x \leq 0$, we have

$$
f_{\Delta}(x)=f\left(1 / \mu^{-1}(h(\Delta))\right)>0 \text { and } g_{\Delta}(x)=0 .
$$

Therefore,

$$
x f_{\Delta}(x)+\frac{p-1}{2}\left|V(y) g_{\Delta}(x)\right|^{2} \leq 0 \leq K_{1}\left(1+|x|^{2}\right) .
$$

Finally, for $x, y \in \mathbb{R}$ with $x>\mu^{-1}(h(\Delta))$, we have

$$
\begin{aligned}
x f_{\Delta}(x)+\frac{p-1}{2}\left|V(y) g_{\Delta}(x)\right|^{2} & \leq x f\left(1 / \mu^{-1}(h(\Delta)) \vee \mu^{-1}(h(\Delta))\right)+\frac{p-1}{2}\left|V(y) g\left(\mu^{-1}(h(\Delta))\right)\right|^{2} \\
& \leq \mu^{-1}(h(\Delta)) f\left(\mu^{-1}(h(\Delta))\right)+\frac{p-1}{2} \mid V(y) g\left(\left.\mu^{-1}(h(\Delta))\right|^{2}\right. \\
& +\left(\frac{x}{\mu^{-1}(h(\Delta))}-1\right) \mu^{-1}(h(\Delta)) f\left(\mu^{-1}(h(\Delta))\right) \\
& \leq K_{1}\left(1+\left[\mu^{-1}(h(\Delta))\right]^{2}\right)+\left(\frac{x}{\mu^{-1}(h(\Delta))}-1\right) \mu^{-1}(h(\Delta)) f\left(\mu^{-1}(h(\Delta))\right),
\end{aligned}
$$

where (23) with $K_{1}$ independent of $\Delta$ has been used. But once again we see from (23) that $x f(x) \leq$ $K_{1}\left(1+|x|^{2}\right)$ for any $x \in \mathbb{R}_{+}$. We therefore have

$$
\begin{aligned}
x f_{\Delta}(x)+\frac{p-1}{2}\left|V(y) g_{\Delta}(x)\right|^{2} & \leq K_{1}\left(1+\left[\mu^{-1}(h(\Delta))\right]^{2}\right)+\left(\frac{x}{\mu^{-1}(h(\Delta))}-1\right) K_{1}\left(1+\left[\mu^{-1}(h(\Delta))\right]^{2}\right) \\
& \leq \frac{x}{\mu^{-1}(h(\Delta))} K_{1}\left(1+\left[\mu^{-1}(h(\Delta))\right]^{2}\right) \\
& \leq x \cdot K_{1}\left(1 / 2+\mu^{-1}(h(\Delta))\right) \\
& \leq x \cdot K_{1}(1 / 2+x) \leq 2 K_{1}\left(1+|x|^{2}\right) .
\end{aligned}
$$

It is worthwhile to note that $\bar{K}=\left(K_{1} \vee K_{2}\right)$. 
From now on, we will let the step size $\Delta \in(0,1)$ be a fraction of $\tau$. That is, we will use $\Delta=\tau / N$ for sufficiently large integer $N$. Let form the discrete-time truncated approximation for SDDE (5). Define $t_{k}=k \Delta$ for $k=-N,-(N-1), . ., 0,1,2, \ldots$ Set $X_{\Delta}\left(t_{k}\right) \approx \xi\left(t_{k}\right)$ for $k=-N,-(N-1), . ., 0$ and form

$$
X_{\Delta}\left(t_{k+1}\right)=X_{\Delta}\left(t_{k}\right)+f_{\Delta}\left(X_{\Delta}\left(t_{k}\right)\right) \Delta+V\left(X_{\Delta}\left(t_{k-N}\right)\right) g_{\Delta}\left(X_{\Delta}\left(t_{k}\right)\right) \Delta B_{k}
$$

for $k=0,1,2 \ldots$, where $\Delta B_{k}=B\left(t_{k+1}\right)-B\left(t_{k}\right)$. Let us now form two versions of the continuous-time truncated EM solutions. The first is defined by

$$
\bar{x}_{\Delta}(t)=\sum_{k=-N}^{\infty} X_{\Delta}\left(t_{k}\right) 1_{[k \Delta,(k+1) \Delta]}(t) .
$$

This is the continuous-time step-process $\bar{x}_{\Delta}(t)$ on $t \in[-\tau, \infty]$, where $1_{[k \Delta,(k+1) \Delta]}$ is the indicator function on $[k \Delta,(k+1) \Delta]$. The other is the continuous-time continuous process $x_{\Delta}(t)$ on $t \in[-\tau, \infty]$ defined by setting $x_{\Delta}(t)=\xi(t)$ for $t \in[-\tau, 0]$ while for $t \geq 0$

$$
x_{\Delta}(t)=\xi(0)+\int_{0}^{t} f_{\Delta}\left(\bar{x}_{\Delta}(s)\right) d s+\int_{0}^{t} V\left(\bar{x}_{\Delta}(s-\tau)\right) g_{\Delta}\left(\bar{x}_{\Delta}(s)\right) d B(s) .
$$

We see that $x_{\Delta}(t)$ is an Itô process on $t \geq 0$ with its Itô differential

$$
d x_{\Delta}(t)=f_{\Delta}\left(\bar{x}_{\Delta}(t)\right) d t+V\left(\bar{x}_{\Delta}(t-\tau)\right) g_{\Delta}\left(\bar{x}_{\Delta}(t)\right) d B(t) .
$$

We can clearly observe that $x_{\Delta}\left(t_{k}\right)=\bar{x}_{\Delta}\left(t_{k}\right)=X_{\Delta}\left(t_{k}\right)$ for all $k=-N,-(N-1), \ldots$ We would like to point out that this numerical scheme is not positivity-preserving. This will however be tackled elsewhere.

\section{Numerical properties of the truncated EM solution}

\subsection{Moment bound of the truncated EM solution}

To upper bound the pth moment of the truncated EM solution, we require the following lemma which shows $x_{\Delta}(t)$ and $\bar{x}_{\Delta}(t)$ are close to each other in $L^{p}$ sense.

Lemma 4.1. Let Assumption 2.1 hold. For any fixed $\Delta \in\left(0, \Delta^{*}\right]$ and $p \geq 2$, we have that

$$
\mathbb{E}\left|x_{\Delta}(t)-\bar{x}_{\Delta}(t)\right|^{p} \leq C_{p} \Delta^{p / 2}(h(\Delta))^{p}, \quad \forall t \geq 0,
$$

where $C_{p}$ stands for generic positive real constants dependent only on $p$ and may change between occurrences. Consequently,

$$
\lim _{\Delta \rightarrow 0} \mathbb{E}\left|x_{\Delta}(t)-\bar{x}_{\Delta}(t)\right|^{p}=0, \quad \forall t \geq 0 .
$$

Proof. Fix any $\Delta \in\left(0, \Delta^{*}\right)$ and $t \geq 0$. There exists an integer $k \geq 0$ such that $t_{k} \leq t \leq t_{k+1}$. By elementary inequality and (26), we obtain from (30) that

$$
\begin{aligned}
\mathbb{E}\left|x_{\Delta}(t)-\bar{x}_{\Delta}(t)\right|^{p} & \leq 2^{p-1}\left(\mathbb{E}\left|\int_{t_{k}}^{t} f_{\Delta}\left(\bar{x}_{\Delta}(s)\right) d s\right|^{p}+\mathbb{E}\left|\int_{t_{k}}^{t} V\left(\bar{x}_{\Delta}(s-\tau)\right) g_{\Delta}\left(\bar{x}_{\Delta}(s)\right) B(s)\right|^{p}\right) \\
& \leq 2^{p-1}\left(\Delta^{p-1} \mathbb{E} \int_{t_{k}}^{t}\left|f_{\Delta}\left(\bar{x}_{\Delta}(s)\right)\right|^{p} d s+\bar{c}_{p} \Delta^{(p-2) / 2} \mathbb{E} \int_{t_{k}}^{t}\left|V\left(\bar{x}_{\Delta}(s-\tau)\right) g_{\Delta}\left(\bar{x}_{\Delta}(s)\right)\right|^{p} d s\right) \\
& \leq 2^{p-1}\left(\Delta^{p-1} \Delta(h(\Delta))^{p}+\bar{c}_{p} \Delta^{(p-2) / 2} \Delta(\lambda h(\Delta))^{p}\right) \\
& \leq 2^{p-1}\left(1 \vee \bar{c}_{p} \lambda^{p}\right) \Delta^{p / 2}(h(\Delta))^{p} \\
& \leq C_{p} \Delta^{p / 2}(h(\Delta))^{p}
\end{aligned}
$$


where $C_{p}=2^{p-1}\left(1 \vee \bar{c}_{p} \lambda^{p}\right)$. Noting from (25) that $\Delta^{p / 2}(h(\Delta))^{p} \leq \Delta^{p / 4}$, we get (33) from (32).

The following lemma reveals the boundedness property of the truncated EM numerical solutions.

Lemma 4.2. Let Assumptions 2.1 and 2.2 hold. Then

$$
\sup _{0 \leq \Delta \leq \Delta^{*}} \sup _{0 \leq t \leq T}\left(\mathbb{E}\left|x_{\Delta}(t)\right|^{p}\right) \leq C_{4}, \quad \forall T>0
$$

where $C_{4}$ stands for generic positive real constants dependent on $T, p, K, \xi$ but independent of $\Delta$ and may change between occurrences.

Proof. Fix any $\Delta \in\left(0, \Delta^{*}\right)$ and $T \geq 0$. By the Itô, we derive from 30 that, for $0 \leq t \leq T$,

$$
\begin{aligned}
\mathbb{E}\left|x_{\Delta}(t)\right|^{p} & \leq|\xi(0)|^{p}+\mathbb{E} \int_{0}^{t} p\left|x_{\Delta}(s)\right|^{p-2}\left(x_{\Delta}(s) f_{\Delta}\left(\bar{x}_{\Delta}(s)\right)+\frac{p-1}{2}\left|V\left(\bar{x}_{\Delta}(s-\tau)\right) g_{\Delta}\left(\bar{x}_{\Delta}(s)\right)\right|^{2}\right) d s \\
& =|\xi(0)|^{p}+\mathbb{E} \int_{0}^{t} p\left|x_{\Delta}(s)\right|^{p-2}\left(\bar{x}_{\Delta}(s) f_{\Delta}\left(\bar{x}_{\Delta}(s)\right)+\frac{p-1}{2}\left|V\left(\bar{x}_{\Delta}(s-\tau)\right) g_{\Delta}\left(\bar{x}_{\Delta}(s)\right)\right|^{2}\right) d s \\
& +\mathbb{E} \int_{0}^{t} p\left|x_{\Delta}(s)\right|^{p-2}\left(x_{\Delta}(s)-\bar{x}_{\Delta}(s)\right) f_{\Delta}\left(\bar{x}_{\Delta}(s)\right) d s
\end{aligned}
$$

By Lemma 3.4 and the Young inequality

$$
a^{p-2} b \leq \frac{p-2}{p} a^{p}+\frac{2}{p} b^{p / 2}, \quad \forall a, b \geq 0,
$$

we then have

$$
\begin{aligned}
\mathbb{E}\left|x_{\Delta}(t)\right|^{p} & \leq|\xi(0)|^{p}+\mathbb{E} \int_{0}^{t} K\left|x_{\Delta}(s)\right|^{p-2}\left(1+\left|\bar{x}_{\Delta}(s)\right|^{2}\right) d s \\
& +(p-2) \mathbb{E} \int_{0}^{t}\left|x_{\Delta}(s)\right|^{p} d s+2 \mathbb{E} \int_{0}^{t}\left|x_{\Delta}(s)-\bar{x}_{\Delta}(s)\right|^{p / 2} \mid f_{\Delta}\left(\left.\bar{x}_{\Delta}(s)\right|^{p / 2} d s\right. \\
& \leq C_{5}+C_{6} \int_{0}^{t}\left(\mathbb{E}\left|x_{\Delta}(s)\right|^{p}+\mathbb{E}\left|\bar{x}_{\Delta}(s)\right|^{p}\right) d s+2 \mathbb{E} \int_{0}^{t}\left|x_{\Delta}(s)-\bar{x}_{\Delta}(s)\right|^{p / 2} \mid f_{\Delta}\left(\left.\bar{x}_{\Delta}(s)\right|^{p / 2} d s\right.
\end{aligned}
$$

where $C_{5}$ and $C_{6}$ are positive constants independent of $\Delta$. By Lemma 4.1 and inequalities 26 and (25), we have

$$
\begin{aligned}
\mathbb{E} \int_{0}^{t}\left|x_{\Delta}(s)-\bar{x}_{\Delta}(s)\right|^{p / 2} \mid f_{\Delta}\left(\left.\bar{x}_{\Delta}(s)\right|^{p / 2} d s\right. & \leq(h(\Delta))^{p / 2} \int_{0}^{T} \mathbb{E}\left(\left|x_{\Delta}(s)-\bar{x}_{\Delta}(s)\right|^{p / 2}\right) d s \\
& \leq(h(\Delta))^{p / 2} \int_{0}^{T}\left(\mathbb{E}\left|x_{\Delta}(s)-\bar{x}_{\Delta}(s)\right|^{p}\right)^{1 / 2} d s \\
& \leq(h(\Delta))^{p / 2} \int_{0}^{T}\left(C_{p} \Delta^{p / 2}(h(\Delta))^{p}\right)^{1 / 2} d s \\
& \leq C_{p} T(h(\Delta))^{p} \Delta^{p / 4} \leq C_{p} T .
\end{aligned}
$$

We therefore have

$$
\begin{aligned}
\mathbb{E}\left|x_{\Delta}(t)\right|^{p} & \leq C_{5}+2 C_{p} T+C_{6} \int_{0}^{t}\left(\mathbb{E}\left|x_{\Delta}(s)\right|^{p}+\mathbb{E}\left|\bar{x}_{\Delta}(s)\right|^{p}\right) d s \\
& \leq C_{5}+2 C_{p} T+2 C_{6} \int_{0}^{t} \sup _{0 \leq u \leq s}\left(\mathbb{E}\left|x_{\Delta}(u)\right|^{p}\right) d s .
\end{aligned}
$$


As this holds for any $t \in[0, T]$ while the right-hand side is non-decreasing in $t$, we then see

$$
\sup _{0 \leq u \leq t}\left(\mathbb{E}\left|x_{\Delta}(u)\right|^{p}\right) \leq C_{5}+2 C_{p} T+2 C_{6} \int_{0}^{t} \sup _{0 \leq u \leq s}\left(\mathbb{E}\left|x_{\Delta}(u)\right|^{p}\right) d s .
$$

The well-known Gronwall inequality gives us

$$
\sup _{0 \leq u \leq T}\left(\mathbb{E}\left|x_{\Delta}(u)\right|^{p}\right) \leq C_{4}
$$

As this holds for any $\Delta \in\left(0, \Delta^{*}\right)$ while $C_{4}=\left(C_{5}+2 C_{p} T\right) e^{2 C_{6} T}$ is independent of $\Delta$, we obtain the required assertion.

\subsection{Strong convergence}

For the numerical solution to converge in finite time to the true solution in $L^{q}$, we need the following condition on the initial data (see, e.g, [23]).

Assumption 4.3. There is a pair of constant $K_{4}>0$ and $\gamma \in(0,1]$ such that for all $-\tau \leq s \leq t \leq 0$, the initial data $\xi$ satisfies

$$
|\xi(t)-\xi(s)| \leq K_{4}|t-s|^{\gamma}
$$

In addition to the above condition, we also need the following lemma.

Lemma 4.4. Let Assumptions 2.1, 2.2, 3.1 and 4.3 hold and $T>0$ be fixed. Then for any $\varepsilon \in(0,1)$, there exists a pair of positive constants $n=n(\varepsilon)$ and $\Delta_{1}=\Delta_{1}(\varepsilon)$ such that

$$
\mathbb{P}\left(\rho_{n} \leq T\right) \leq \varepsilon
$$

for each $\Delta \in\left(0, \Delta_{1}\right]$, where

$$
\rho_{n}=\rho_{n}(\Delta)=\inf \left\{t \in[0, T]: x_{\Delta}(t) \notin(1 / n, n)\right\}
$$

is the stopping time.

Proof. Define a $C^{2}$-function, $H: \mathbb{R}_{+} \rightarrow \mathbb{R}_{+}$by

$$
H(x)=1 / x^{2}+x^{2} .
$$

Clearly, $H(x) \rightarrow \infty$ as $x \rightarrow \infty$ or $x \rightarrow 0$. For $s \in\left[0, t \wedge \rho_{n}\right]$, we can derive from the Itô formula that

$$
\begin{aligned}
\mathbb{E}\left(H\left(x_{\Delta}\left(t \wedge \rho_{n}\right)\right)\right) & =H(\xi(0))+\mathbb{E} \int_{0}^{t \wedge \rho_{n}}\left(H_{x}\left(x_{\Delta}(s)\right) f_{\Delta}\left(\bar{x}_{\Delta}(s)\right)\right. \\
& \left.+\frac{1}{2} H_{x x}\left(x_{\Delta}(s)\right) V\left(\bar{x}_{\Delta}(s-\tau)\right)^{2} g_{\Delta}\left(\bar{x}_{\Delta}(s)\right)^{2}\right) d s
\end{aligned}
$$

But

$$
\begin{aligned}
& H_{x}\left(x_{\Delta}(s)\right) f_{\Delta}\left(\bar{x}_{\Delta}(s)\right)+\frac{1}{2} H_{x x}\left(x_{\Delta}(s)\right) V\left(\bar{x}_{\Delta}(s-\tau)\right)^{2} g_{\Delta}\left(\bar{x}_{\Delta}(s)\right)^{2} \\
& \leq L H\left(x_{\Delta}(s), x_{\Delta}(s-\tau)\right)+H_{x}\left(x_{\Delta}(s)\right)\left(f_{\Delta}\left(\bar{x}_{\Delta}(s)\right)-f_{\Delta}\left(x_{\Delta}(s)\right)\right) \\
& +\frac{1}{2} H_{x x}\left(x_{\Delta}(s)\right)\left(V\left(\bar{x}_{\Delta}(s-\tau)\right)^{2} g_{\Delta}\left(\bar{x}_{\Delta}(s)\right)^{2}-V\left(x_{\Delta}(s-\tau)\right)^{2} g_{\Delta}\left(x_{\Delta}(s)\right)^{2}\right)
\end{aligned}
$$


where $L H$ is (6) with $H$ independent of $t$, defined here by

$$
L H\left(x_{\Delta}(s), x_{\Delta}(s-\tau)\right)=H_{x}\left(x_{\Delta}(s)\right) f_{\Delta}\left(x_{\Delta}(s)\right)+\frac{1}{2} H_{x x}\left(x_{\Delta}(s)\right) V\left(x_{\Delta}(s-\tau)\right)^{2} g_{\Delta}\left(x_{\Delta}(s)\right)^{2} .
$$

By Assumptions 2.1 and 2.2, there exists a constant $K_{3}$ such that

$$
L H\left(x_{\Delta}(s), x_{\Delta}(s-\tau)\right) \leq K_{3}
$$

and

$$
\begin{aligned}
& H_{x}\left(x_{\Delta}(s)\right) f_{\Delta}\left(\bar{x}_{\Delta}(s)\right)+\frac{1}{2} H_{x x}\left(x_{\Delta}(s)\right) V\left(\bar{x}_{\Delta}(s-\tau)\right)^{2} g_{\Delta}\left(\bar{x}_{\Delta}(s)\right)^{2} \\
& \leq K_{3}+H_{x}\left(x_{\Delta}(s)\right)\left(f_{\Delta}\left(\bar{x}_{\Delta}(s)\right)-f_{\Delta}\left(x_{\Delta}(s)\right)\right)+\frac{1}{2} H_{x x}\left(x_{\Delta}(s)\right)\left(V\left(\bar{x}_{\Delta}(s-\tau)\right)^{2} g_{\Delta}\left(\bar{x}_{\Delta}(s)\right)^{2}\right. \\
& \left.-V\left(x_{\Delta}(s-\tau)\right)^{2} g_{\Delta}\left(x_{\Delta}(s)\right)^{2}\right) .
\end{aligned}
$$

Recalling Lemma 3.2 , we have that for $s \in\left[t \wedge \rho_{n}\right]$

$$
\left|f_{\Delta}\left(\bar{x}_{\Delta}(s)\right)-f_{\Delta}\left(x_{\Delta}(s)\right)\right|=K_{n}\left|\bar{x}_{\Delta}(s)-x_{\Delta}(s)\right| .
$$

For any $\bar{x}_{\Delta}(s), x_{\Delta}(s) \in[1 / n, n]$, by (24), $\left|g_{\Delta}\left(\bar{x}_{\Delta}(s)\right)\right| \vee\left|g_{\Delta}\left(x_{\Delta}(s)\right)\right| \leq \mu(n)$. Hence by Lemma 3.2, we have that for $s \in\left[t \wedge \rho_{n}\right]$

$$
\begin{aligned}
\left|g_{\Delta}\left(\bar{x}_{\Delta}(s)\right)^{2}-g_{\Delta}\left(x_{\Delta}(s)\right)^{2}\right| & =\left|g_{\Delta}\left(\bar{x}_{\Delta}(s)\right)-g_{\Delta}\left(x_{\Delta}(s)\right)\right|\left|g_{\Delta}\left(\bar{x}_{\Delta}(s)\right)+g_{\Delta}\left(x_{\Delta}(s)\right)\right| \\
& \leq 2 \mu(n) K_{n}\left|\bar{x}_{\Delta}(s)-x_{\Delta}(s)\right| .
\end{aligned}
$$

Moreover, for $s \in\left[t \wedge \rho_{n}\right]$, we obtain from Assumptions 2.1 and 3.1 that

$$
\begin{aligned}
\left|V\left(\bar{x}_{\Delta}(s-\tau)\right)^{2}-V\left(x_{\Delta}(s-\tau)\right)^{2}\right| & =\left|V\left(\bar{x}_{\Delta}(s-\tau)\right)-V\left(x_{\Delta}(s-\tau)\right)\right|\left|V\left(\bar{x}_{\Delta}(s-\tau)\right)+V\left(x_{\Delta}(s-\tau)\right)\right| \\
& \leq 2 \lambda L_{n}\left|\bar{x}_{\Delta}(s-\tau)-x_{\Delta}(s-\tau)\right|
\end{aligned}
$$

Consequently,

$$
\begin{aligned}
& V\left(\bar{x}_{\Delta}(s-\tau)\right)^{2} g_{\Delta}\left(\bar{x}_{\Delta}(s)\right)^{2}-V\left(x_{\Delta}(s-\tau)\right)^{2} g_{\Delta}\left(x_{\Delta}(s)\right)^{2}=V\left(\bar{x}_{\Delta}(s-\tau)\right)^{2} g_{\Delta}\left(\bar{x}_{\Delta}(s)\right)^{2} \\
& -V\left(\bar{x}_{\Delta}(s-\tau)\right)^{2} g_{\Delta}\left(x_{\Delta}(s)\right)^{2}+V\left(\bar{x}_{\Delta}(s-\tau)\right)^{2} g_{\Delta}\left(x_{\Delta}(s)\right)^{2}-V\left(x_{\Delta}(s-\tau)\right)^{2} g_{\Delta}\left(x_{\Delta}(s)\right)^{2} \\
& =g_{\Delta}\left(x_{\Delta}(s)\right)^{2}\left(V\left(\bar{x}_{\Delta}(s-\tau)\right)^{2}-V\left(x_{\Delta}(s-\tau)\right)^{2}\right)+V\left(\bar{x}_{\Delta}(s-\tau)\right)^{2}\left(g_{\Delta}\left(\bar{x}_{\Delta}(s)\right)^{2}-g_{\Delta}\left(x_{\Delta}(s)\right)^{2}\right) \\
& \leq 2 \lambda(\mu(n))^{2} L_{n}\left|\bar{x}_{\Delta}(s-\tau)-x_{\Delta}(s-\tau)\right|+2 \lambda^{2} \mu(n) K_{n}\left|\bar{x}_{\Delta}(s)-x_{\Delta}(s)\right| .
\end{aligned}
$$

So we have

$$
\begin{aligned}
& H_{x}\left(x_{\Delta}(s)\right) f_{\Delta}\left(\bar{x}_{\Delta}(s)\right)+\frac{1}{2} H_{x x}\left(x_{\Delta}(s)\right) V\left(\bar{x}_{\Delta}(s-\tau)\right)^{2} g_{\Delta}\left(\bar{x}_{\Delta}(s)\right)^{2} \\
& \leq K_{3}+\lambda(\mu(n))^{2} L_{n} H_{x x}\left(x_{\Delta}(s)\right)\left|\bar{x}_{\Delta}(s-\tau)-x_{\Delta}(s-\tau)\right| \\
& +\left(K_{n} H_{x}\left(x_{\Delta}(s)\right)+\lambda^{2} K_{n} \mu(n) H_{x x}\left(x_{\Delta}(s)\right)\right)\left|\bar{x}_{\Delta}(s)-x_{\Delta}(s)\right| \\
& \leq K_{3}+\zeta_{n}\left|\bar{x}_{\Delta}(s-\tau)-x_{\Delta}(s-\tau)\right|+\zeta_{n}^{*}\left|\bar{x}_{\Delta}(s)-x_{\Delta}(s)\right|,
\end{aligned}
$$

where

$$
\zeta_{n}=\max _{1 / n \leq x \leq n}\left[\lambda(\mu(n))^{2} L_{n} H_{x x}(x)\right]
$$


and

$$
\zeta_{n}^{*}=\max _{1 / n \leq x \leq n}\left[K_{n} H_{x}(x)+\lambda^{2} K_{n} \mu(n) H_{x x}(x)\right] .
$$

We now have

$$
\begin{aligned}
\mathbb{E}\left(H\left(x_{\Delta}\left(t \wedge \rho_{n}\right)\right)\right) & \leq H(\xi(0))+\mathbb{E} \int_{0}^{t \wedge \rho_{n}}\left(K_{3}+\zeta_{n}\left|\bar{x}_{\Delta}(s-\tau)-x_{\Delta}(s-\tau)\right|+\zeta_{n}^{*}\left|\bar{x}_{\Delta}(s)-x_{\Delta}(s)\right|\right) d s \\
& \leq H(\xi(0))+K_{3} T+\zeta_{n} \mathbb{E} \int_{-\tau}^{0}|\xi([s / \Delta] \Delta)-\xi(s)| d s+\left(\zeta_{n}+\zeta_{n}^{*}\right) \int_{0}^{T} \mathbb{E}\left|x_{\Delta}(s)-\bar{x}_{\Delta}(s)\right| d s \\
& \leq H(\xi(0))+K_{3} T+\zeta_{n} K_{4} \Delta^{\gamma} \tau+\left(\zeta_{n}+\zeta_{n}^{*}\right) \int_{0}^{T}\left(\mathbb{E}\left|x_{\Delta}(s)-\bar{x}_{\Delta}(s)\right|^{p}\right)^{1 / p} d s .
\end{aligned}
$$

By Lemma 4.1 and (25), we obtain

$$
\mathbb{E}\left(H\left(x_{\Delta}\left(t \wedge \rho_{n}\right)\right)\right) \leq H(\xi(0))+K_{3} T+\zeta_{n} K_{4} \Delta^{\gamma} \tau+\left(\zeta_{n}+\zeta_{n}^{*}\right) T C_{p}^{1 / p} \Delta^{1 / 4} .
$$

Therefore

$$
\mathbb{P}\left(\rho_{n} \leq T\right) \leq \frac{H(\xi(0))+K_{3} T+\zeta_{n} K_{4} \Delta^{\gamma} \tau+\left(\zeta_{n}+\zeta_{n}^{*}\right) T C_{p}^{1 / p} \Delta^{1 / 4}}{H(1 / n) \wedge H(n)}
$$

For $\varepsilon \in(0,1)$, we may choose sufficiently large $n$ such that

$$
\frac{H(\xi(0))+K_{3} T}{H(1 / n) \wedge H(n)} \leq \frac{\varepsilon}{2}
$$

and sufficiently small step size $\Delta \in\left(0, \Delta_{1}\right]$ such that

$$
\frac{\zeta_{n} K_{4} \Delta^{\gamma} \tau+\left(\zeta_{n}+\zeta_{n}^{*}\right) T C_{p}^{1 / p} \Delta^{1 / 4}}{H(1 / n) \wedge H(n)} \leq \frac{\varepsilon}{2} .
$$

Combining (40) and (41), we get the required assertion.

To establish the strong convergence of the truncated EM scheme, we first define the stopping time

$$
v_{n}=\tau_{n} \wedge \rho_{n},
$$

where $\tau_{n}$ and $\rho_{n}$ are $(11)$ and $(36)$ respectively.

Lemma 4.5. Let Assumptions 2.1, 3.1 and 4.3 hold. Then, for any $p \geq 2, T>0$ and sufficiently large $n$

$$
\mathbb{E}\left(\sup _{0 \leq t \leq T}\left|x_{\Delta}\left(t \wedge v_{n}\right)-x\left(t \wedge v_{n}\right)\right|^{p}\right) \leq\left(C_{1}(n, p, T)+C_{2}(n, p, T)\right) \Delta^{p(1 / 4 \wedge \gamma)} e^{C_{3}(n, p, T)}
$$

and

$$
\lim _{\Delta \rightarrow 0} \mathbb{E}\left(\sup _{0 \leq t \leq T}\left|x_{\Delta}\left(t \wedge v_{n}\right)-x\left(t \wedge v_{n}\right)\right|^{p}\right)=0
$$

where

$$
\begin{gathered}
C_{1}(n, p, T)=4^{p-1} T^{\frac{p-2}{2}} C(p) L_{n} K_{4}^{p} \tau(\mu(n))^{p}, \\
C_{2}(n, p, T)=8^{p-1} C_{p}\left(T^{\frac{p}{2}}(\mu(n))^{p} C(p) L_{n}+T^{\frac{p}{2}} \lambda^{p} C(p) K_{n}+2^{1-p} T^{p}\right)
\end{gathered}
$$

and

$$
C_{3}(n, p, T)=8^{p-1}\left(T^{\frac{p-2}{2}}(\mu(n))^{p} C(p) L_{n}+T^{\frac{p-2}{2}} \lambda^{p} C(p) K_{n}+2^{1-p} T^{p-1}\right) .
$$


Proof. It follows from (5) and (31) that

$$
\begin{aligned}
{\left[x_{\Delta}\left(t \wedge v_{n}\right)-x\left(t \wedge v_{n}\right)\right] } & =\int_{0}^{t \wedge v_{n}}\left[f_{\Delta}\left(\bar{x}_{\Delta}(s)\right)-f(x(s))\right] d s+\int_{0}^{t \wedge v_{n}}\left[V\left(\bar{x}_{\Delta}(s-\tau)\right) g_{\Delta}\left(\bar{x}_{\Delta}(s)\right)\right. \\
& -V(x(s-\tau)) g(x(s))] d B(s) .
\end{aligned}
$$

Applying the inequality $|a+b|^{p} \leq 2^{p-1}\left(|a|^{p}+|b|^{p}\right)$ yields

$$
\begin{aligned}
\left|x_{\Delta}\left(t \wedge v_{n}\right)-x\left(t \wedge v_{n}\right)\right|^{p} & \leq 2^{p-1}\left(\left|\int_{0}^{t \wedge v_{n}}\left[f_{\Delta}\left(\bar{x}_{\Delta}(s)\right)-f(x(x))\right] d s\right|^{p}+\mid \int_{0}^{t \wedge v_{n}}\left[V\left(\bar{x}_{\Delta}(s-\tau)\right) g_{\Delta}\left(\bar{x}_{\Delta}(s)\right)\right.\right. \\
& \left.-V(x(s-\tau)) g(x(s))]\left.d B(s)\right|^{p}\right)
\end{aligned}
$$

For $t_{1} \in[0, T]$

$$
\begin{aligned}
\mathbb{E}\left(\sup _{0 \leq t \leq t_{1}}\left|x_{\Delta}\left(t \wedge v_{n}\right)-x\left(t \wedge v_{n}\right)\right|^{p}\right) & \leq 2^{p-1}\left(\mathbb{E}\left|\int_{0}^{t_{1} \wedge v_{n}}\left[f_{\Delta}\left(\bar{x}_{\Delta}(s)\right)-f(x(s))\right] d s\right|^{p}\right. \\
& +\mathbb{E}\left(\sup _{0 \leq t \leq t_{1}} \mid \int_{0}^{t_{1} \wedge v_{n}}\left[V\left(\bar{x}_{\Delta}(s-\tau)\right) g_{\Delta}\left(\bar{x}_{\Delta}(s)\right)\right.\right. \\
& \left.\left.-V(x(s-\tau)) g(x(s))]\left.d B(s)\right|^{p}\right)\right) .
\end{aligned}
$$

By the Hölder inequality

$$
\mathbb{E}\left(\left|\int_{0}^{t_{1} \wedge v_{n}}\left[f_{\Delta}\left(\bar{x}_{\Delta}(s)\right)-f(x(s))\right] d s\right|^{p}\right) \leq T^{p-1} \mathbb{E}\left(\int_{0}^{t_{1} \wedge v_{n}}\left|f_{\Delta}\left(\bar{x}_{\Delta}(s)\right)-f(x(s))\right|^{p} d s\right) .
$$

Also using Theorem 7.2 in [11], we obtain

$$
\begin{aligned}
& \mathbb{E}\left(\sup _{0 \leq t \leq t_{1}}\left|\int_{0}^{t_{1} \wedge v_{n}}\left(V\left(\bar{x}_{\Delta}(s-\tau)\right) g_{\Delta}\left(\bar{x}_{\Delta}(s)\right)-V(x(s-\tau)) g(x(s))\right) d B(s)\right|^{p}\right) \\
& \leq T^{\frac{p-2}{2}} C(p) \mathbb{E}\left(\int_{0}^{t_{1} \wedge v_{n}}\left|V\left(\bar{x}_{\Delta}(s-\tau)\right) g_{\Delta}\left(\bar{x}_{\Delta}(s)\right)-V(x(s-\tau)) g(x(s))\right|^{p} d s\right),
\end{aligned}
$$

where $C(p)$ is a constant. We now have

$$
\begin{aligned}
\mathbb{E}\left(\sup _{0 \leq t \leq t_{1}}\left|x_{\Delta}\left(t \wedge v_{n}\right)-x\left(t \wedge v_{n}\right)\right|^{p}\right) & \leq 2^{p-1}\left(T^{p-1} \mathbb{E} \int_{0}^{t_{1} \wedge v_{n}}\left|f_{\Delta}\left(\bar{x}_{\Delta}(s)\right)-f(x(s))\right|^{p} d s\right. \\
& +T^{\frac{p-2}{2}} C(p) \mathbb{E} \int_{0}^{t_{1} \wedge v_{n}} \mid V\left(\bar{x}_{\Delta}(s-\tau)\right) g_{\Delta}\left(\bar{x}_{\Delta}(s)\right) \\
& \left.-\left.V(x(s-\tau)) g(x(s))\right|^{p} d s\right) .
\end{aligned}
$$

Meanwhile

$$
\begin{aligned}
& \mathbb{E} \int_{0}^{t_{1} \wedge v_{n}}\left(\left|V\left(\bar{x}_{\Delta}(s-\tau)\right) g_{\Delta}\left(\bar{x}_{\Delta}(s)\right)-V(x(s-\tau)) g(x(s))\right|^{p}\right) d s \\
& =\mathbb{E} \int_{0}^{t_{1} \wedge v_{n}}\left(\mid V\left(\bar{x}_{\Delta}(s-\tau)\right) g_{\Delta}\left(\bar{x}_{\Delta}(s)\right)-V(x(s-\tau)) g_{\Delta}\left(\bar{x}_{\Delta}(s)\right)+V(x(s-\tau)) g_{\Delta}\left(\bar{x}_{\Delta}(s)\right)\right. \\
& \left.-\left.V(x(s-\tau)) g(x(s))\right|^{p}\right) d s .
\end{aligned}
$$


By elementary inequality,

$$
\begin{aligned}
& \mathbb{E} \int_{0}^{t_{1} \wedge v_{n}}\left(\left|V\left(\bar{x}_{\Delta}(s-\tau)\right) g_{\Delta}\left(\bar{x}_{\Delta}(s)\right)-V(x(s-\tau)) g(x(s))\right|^{p}\right) d s \\
& \leq 2^{p-1} \mathbb{E} \int_{0}^{t_{1} \wedge v_{n}}\left(\left|V\left(\bar{x}_{\Delta}(s-\tau)\right) g_{\Delta}\left(\bar{x}_{\Delta}(s)\right)-V(x(s-\tau)) g_{\Delta}\left(\bar{x}_{\Delta}(s)\right)\right|^{p}\right. \\
& \left.+\left|V(x(s-\tau)) g_{\Delta}\left(\bar{x}_{\Delta}(s)\right)-V(x(s-\tau)) g(x(s))\right|^{p}\right) d s \\
& \left.\leq 2^{p-1} \mathbb{E} \int_{0}^{t_{1} \wedge v_{n}} g_{\Delta}\left(\bar{x}_{\Delta}(s)\right)^{p}\left|V\left(\bar{x}_{\Delta}(s-\tau)\right)-V(x(s-\tau))\right|^{p}+V(x(s-\tau))^{p}\left|g_{\Delta}\left(\bar{x}_{\Delta}(s)\right)-g(x(s))\right|^{p}\right) d s
\end{aligned}
$$

By (8)

$$
\begin{aligned}
& \mathbb{E} \int_{0}^{t_{1} \wedge v_{n}}\left(\left|V\left(\bar{x}_{\Delta}(s-\tau)\right) g_{\Delta}\left(\bar{x}_{\Delta}(s)\right)-V(x(s-\tau)) g(x(s))\right|^{p} d s\right) \\
& \leq 2^{p-1} \mathbb{E} \int_{0}^{t_{1} \wedge v_{n}} g_{\Delta}\left(\bar{x}_{\Delta}(s)\right)^{p}\left|V\left(\bar{x}_{\Delta}(s-\tau)\right)-V(x(s-\tau))\right|^{p} d s+2^{p-1} \lambda^{p} \mathbb{E} \int_{0}^{t_{1} \wedge v_{n}}\left|g_{\Delta}\left(\bar{x}_{\Delta}(s)\right)-g(x(s))\right|^{p} d s .
\end{aligned}
$$

Moreover, by (24), for any $\bar{x}_{\Delta}(s) \in[1 / n, n]$, clearly $\left|g_{\Delta}\left(\bar{x}_{\Delta}(s)\right)\right| \leq \mu(n)$. Hence

$$
\begin{aligned}
& \mathbb{E} \int_{0}^{t_{1} \wedge v_{n}}\left(\left|V\left(\bar{x}_{\Delta}(s-\tau)\right) g_{\Delta}\left(\bar{x}_{\Delta}(s)\right)-V(x(s-\tau)) g(x(s))\right|^{p} d s\right) \\
& \leq 2^{p-1}(\mu(n))^{p} \mathbb{E} \int_{0}^{t_{1} \wedge v_{n}}\left|V\left(\bar{x}_{\Delta}(s-\tau)\right)-V(x(s-\tau))\right|^{p} d s+2^{p-1} \lambda^{p} \mathbb{E} \int_{0}^{t_{1} \wedge v_{n}}\left|g_{\Delta}\left(\bar{x}_{\Delta}(s)\right)-g(x(s))\right|^{p} d s .
\end{aligned}
$$

We note from Assumption 3.1 that

$$
\left|V\left(\bar{x}_{\Delta}(s-\tau)\right)-V(x(s-\tau))\right|^{p} \leq L_{n}\left|\bar{x}_{\Delta}(s-\tau)-x(s-\tau)\right|^{p} .
$$

So by Assumption 4.3 , for $s \in\left[t_{1} \wedge v_{n}\right]$ we obtain

$$
\begin{aligned}
\mathbb{E} \int_{0}^{t_{1} \wedge v_{n}}\left|V\left(\bar{x}_{\Delta}(s-\tau)\right)-V(x(s-\tau))\right|^{p} d s & \leq L_{n} \mathbb{E} \int_{0}^{t_{1} \wedge v_{n}}\left|\bar{x}_{\Delta}(s-\tau)-x(s-\tau)\right|^{p} d s \\
& \leq L_{n} \mathbb{E} \int_{-\tau}^{0}|\xi([s / \Delta] \Delta)-\xi(s)|^{p} d s+L_{n} \mathbb{E} \int_{0}^{t_{1} \wedge v_{n}}\left|\bar{x}_{\Delta}(s)-x(s)\right|^{p} d s \\
& \leq L_{n} K_{4}^{p} \Delta^{p \gamma} \tau+L_{n} \mathbb{E} \int_{0}^{t_{1} \wedge v_{n}}\left|\bar{x}_{\Delta}(s)-x(s)\right|^{p} d s
\end{aligned}
$$

We now have

$$
\begin{aligned}
\mathbb{E}\left(\sup _{0 \leq t \leq t_{1}}\left|x_{\Delta}\left(t \wedge v_{n}\right)-x\left(t \wedge v_{n}\right)\right|^{p}\right) & \leq 2^{p-1} T^{p-1} \mathbb{E} \int_{0}^{t_{1} \wedge v_{n}} \mid f_{\Delta}\left(\bar{x}_{\Delta}(s)\right)-f\left(\left.x(s)\right|^{p} d s\right. \\
& +4^{p-1} T^{\frac{p-2}{2}}(\mu(n))^{p} C(p) L_{n} K_{4}^{p} \Delta^{p \gamma} \tau \\
& +4^{p-1} T^{\frac{p-2}{2}}(\mu(n))^{p} C(p) L_{n} \mathbb{E} \int_{0}^{t_{1} \wedge v_{n}}\left|\bar{x}_{\Delta}(s)-x(s)\right|^{p} d s \\
& +4^{p-1} T^{\frac{p-2}{2}} \lambda^{p} C(p) \mathbb{E} \int_{0}^{t_{1} \wedge v_{n}}\left|g_{\Delta}\left(\bar{x}_{\Delta}(s)\right)-g(x(s))\right|^{p} d s .
\end{aligned}
$$


We note from the definition of the truncated functions $f_{\Delta}$ and $g_{\Delta}$ that

$$
f_{\Delta}\left(\bar{x}_{\Delta}(s)\right)=f\left(\bar{x}_{\Delta}(s)\right) \text { and } g_{\Delta}\left(\bar{x}_{\Delta}(s)\right)=g\left(\bar{x}_{\Delta}(s)\right)
$$

for $s \in\left[t_{1} \wedge v_{n}\right]$. Hence by Lemma 3.2, we have

$$
\left|f\left(\bar{x}_{\Delta}(s)\right)-f(x(s))\right|^{p} \vee\left|g\left(\bar{x}_{\Delta}(s)\right)-g(x(s))\right|^{p} \leq K_{n}\left|\bar{x}_{\Delta}(s)-x(s)\right|^{p}
$$

for $s \in\left[t_{1} \wedge v_{n}\right]$. This implies

$$
\begin{aligned}
\mathbb{E}\left(\sup _{0 \leq t \leq t_{1}}\left|x_{\Delta}\left(t \wedge v_{n}\right)-x\left(t \wedge v_{n}\right)\right|^{p}\right) \leq & 4^{p-1} T^{\frac{p-2}{2}} C(p) L_{n} K_{4}^{p}(\mu(n))^{p} \Delta^{p \gamma} \tau \\
& +2^{p-1} T^{p-1} K_{n} \mathbb{E} \int_{0}^{t_{1} \wedge v_{n}}\left|\bar{x}_{\Delta}(s)-x(s)\right|^{p} d s \\
& +4^{p-1} T^{\frac{p-2}{2}}(\mu(n))^{p} C(p) L_{n} \mathbb{E} \int_{0}^{t_{1} \wedge v_{n}}\left|\bar{x}_{\Delta}(s)-x(s)\right|^{p} d s \\
& +4^{p-1} T^{\frac{p-2}{2}} \lambda^{p} C(p) K_{n} \mathbb{E} \int_{0}^{t_{1} \wedge v_{n}}\left|\bar{x}_{\Delta}(s)-x(s)\right|^{p} d s .
\end{aligned}
$$

This yields

$$
\begin{aligned}
\mathbb{E}\left(\sup _{0 \leq t \leq t_{1}}\left|x_{\Delta}\left(t \wedge v_{n}\right)-x\left(t \wedge v_{n}\right)\right|^{p}\right) \leq & 4^{p-1} T^{\frac{p-2}{2}} C(p) L_{n} K_{4}^{p}(\mu(n))^{p} \Delta^{p \gamma} \tau \\
& +4^{p-1}\left(T^{\frac{p-2}{2}}(\mu(n))^{p} C(p) L_{n}+T^{\frac{p-2}{2}} \lambda^{p} C(p) K_{n}+2^{1-p} T^{p-1} K_{n}\right) \\
& \times \mathbb{E} \int_{0}^{t_{1} \wedge v_{n}}\left|\bar{x}_{\Delta}(s)-x(s)\right|^{p} d s .
\end{aligned}
$$

Using elementary inequality, we have

$$
\begin{aligned}
\mathbb{E} \int_{0}^{t_{1} \wedge v_{n}}\left|\bar{x}_{\Delta}(s)-x(s)+x_{\Delta}(s)-x_{\Delta}(s)\right|^{p} d s & \leq 2^{p-1} \mathbb{E}\left(\int_{0}^{t_{1} \wedge v_{n}}\left(\left|\bar{x}_{\Delta}(s)-x_{\Delta}(s)\right|^{p}+\left|x_{\Delta}(s)-x(s)\right|^{p}\right) d s\right) \\
& \leq 2^{p-1}\left(\int_{0}^{T} \mathbb{E}\left|\bar{x}_{\Delta}(s)-x_{\Delta}(s)\right|^{p} d s+\mathbb{E} \int_{0}^{t_{1}} \sup _{0 \leq t \leq T} \mid x_{\Delta}\left(t \wedge v_{n}\right)\right. \\
& \left.-\left.x\left(t \wedge v_{n}\right)\right|^{p} d s\right) .
\end{aligned}
$$

This implies

$$
\begin{aligned}
& \mathbb{E}\left(\sup _{0 \leq t \leq t_{1}}\left|x_{\Delta}\left(t \wedge v_{n}\right)-x\left(t \wedge v_{n}\right)\right|^{p}\right) \\
& \leq 4^{p-1} T^{\frac{p-2}{2}} C(p) L_{n} K_{4}^{p} \tau(\mu(n))^{p} \Delta^{p \gamma} \\
& +8^{p-1}\left(T^{\frac{p-2}{2}}(\mu(n))^{p} C(p) L_{n}+T^{\frac{p-2}{2}} \lambda^{p} C(p) K_{n}+2^{1-p} T^{p-1} K_{n}\right) \\
& \times\left(\int_{0}^{T} \mathbb{E}\left|\bar{x}_{\Delta}(s)-x_{\Delta}(s)\right|^{p} d s+\mathbb{E} \int_{0}^{t_{1}} \sup _{0 \leq t \leq T}\left|x_{\Delta}\left(t \wedge v_{n}\right)-x\left(t \wedge v_{n}\right)\right|^{p} d s\right) .
\end{aligned}
$$


By Lemma 4.1

$$
\begin{aligned}
& \mathbb{E}\left(\sup _{0 \leq t \leq t_{1}}\left|x_{\Delta}\left(t \wedge v_{n}\right)-x\left(t \wedge v_{n}\right)\right|^{p}\right) \\
& \leq 4^{p-1} T^{\frac{p-2}{2}} C(p) L_{n} K_{4}^{p} \tau(\mu(n))^{p} \Delta^{p \gamma} \\
& +8^{p-1} C_{p}\left(T^{\frac{p}{2}}(\mu(n))^{p} C(p) L_{n}+T^{\frac{p}{2}} \lambda^{p} C(p) K_{n}+2^{1-p} T^{p}\right) \Delta^{p / 4} \\
& +8^{p-1}\left(T^{\frac{p-2}{2}}(\mu(n))^{p} C(p) L_{n}+T^{\frac{p-2}{2}} \lambda^{p} C(p) K_{n}+2^{1-p} T^{p-1}\right) \mathbb{E} \int_{0}^{t_{1}} \sup _{0 \leq t \leq T}\left|x_{\Delta}\left(t \wedge v_{n}\right)-x\left(t \wedge v_{n}\right)\right|^{p} d s
\end{aligned}
$$

By the Grownwall inequality, we obtain

$$
\mathbb{E}\left(\sup _{0 \leq t \leq T}\left|x_{\Delta}\left(t \wedge v_{n}\right)-x\left(t \wedge v_{n}\right)\right|^{p}\right) \leq\left(C_{1}(n, p, T)+C_{2}(n, p, T)\right) \Delta^{p(1 / 4 \wedge \gamma)} e^{C_{3}(n, p, T)}
$$

as the required assertion. Moreover, we obtain (44) by letting $\Delta \rightarrow 0$.

Theorem 4.6. Let Assumptions 2.1, 2.2, 3.1 and 4.3 hold. Then, for $q \in[2, p)$

$$
\lim _{\Delta \rightarrow 0} \mathbb{E}\left(\sup _{0 \leq t \leq T}\left|x_{\Delta}(t)-x(t)\right|^{q}\right)=0
$$

Proof. Let $v_{n}$ be the same as before. Set

$$
e_{\Delta}(t)=x_{\Delta}(t)-x(t) .
$$

Clearly

$$
\mathbb{E}\left(\sup _{0 \leq t \leq T}\left|e_{\Delta}(t)\right|^{q}\right)=\mathbb{E}\left(\sup _{0 \leq t \leq T}\left|e_{\Delta}(t)\right|^{q} 1_{\left\{\tau_{n}>T \text { and } \rho_{n}>T\right\}}\right)+\mathbb{E}\left(\sup _{0 \leq t \leq T}\left|e_{\Delta}(t)\right|^{q} 1_{\left\{\tau_{n} \leq T \text { or } \rho_{n} \leq T\right\}}\right) .
$$

For any arbitrary $\varrho \geq 0$, the Young inequality

$$
\left(\varrho a^{p}\right)^{q / p}\left(\frac{b^{(p / p-q)}}{\varrho^{(q / p-q)}}\right)^{(p-q / p)} \leq \frac{q \varrho}{p} a^{p}+\frac{p-q}{p \varrho^{(q / p-q)}} b^{(p / p-q)}, \quad \forall a, b>0,
$$

gives us

$$
\mathbb{E}\left(\left|e_{\Delta}(t)\right|^{q}\right) 1_{\left\{\tau_{n} \leq T \text { or } \rho_{n} \leq T\right\}} \leq \frac{q \varrho}{p} \mathbb{E}\left(\sup _{0 \leq t \leq T}\left|e_{\Delta}(t)\right|^{p}\right)+\frac{p-q}{p \varrho^{q /(p-q)}} \mathbb{P}\left(\tau_{n} \leq T \text { or } \rho_{n} \leq T\right) .
$$

Consequently,

$$
\begin{aligned}
\mathbb{E}\left(\sup _{0 \leq t \leq T}\left|e_{\Delta}(t)\right|^{q}\right) & \leq \mathbb{E}\left(\sup _{0 \leq t \leq T}\left|e_{\Delta}(t)\right|^{q} 1_{\left\{\tau_{n}>T \text { and } \rho_{n}>T\right\}}\right)+\frac{q \varrho}{p} \mathbb{E}\left(\sup _{0 \leq t \leq T}\left|e_{\Delta}(t)\right|^{p}\right) \\
& +\frac{p-q}{p \varrho^{q /(p-q)}} \mathbb{P}\left(\tau_{n} \leq T \text { or } \rho_{n} \leq T\right) .
\end{aligned}
$$

By elementary inequality, we can derive to obtain

$$
\begin{aligned}
\mathbb{E}\left(\sup _{0 \leq t \leq T}\left|e_{\Delta}(t)\right|^{p}\right) & \leq 2^{p-1} \mathbb{E}\left(\sup _{0 \leq t \leq T}\left(\left|x_{\Delta}(t)\right|^{p}+|x(t)|^{p}\right)\right) \\
& \leq 2^{p} \mathbb{E}\left(\sup _{0 \leq t \leq T}\left(\left|x_{\Delta}(t)\right|^{p}\right) \vee \sup _{0 \leq t \leq T}\left(|x(t)|^{p}\right)\right) .
\end{aligned}
$$


So by Lemmas 2.4 and 4.2

$$
\mathbb{E}\left(\sup _{0 \leq t \leq T}\left|e_{\Delta}(t)\right|^{p}\right) \leq 2^{p}\left(C_{1} \vee C_{4}\right)
$$

Moreover,

$$
\begin{aligned}
\mathbb{E}\left(\sup _{0 \leq t \leq T}\left|e_{\Delta}(t)\right|^{p} 1_{\left\{\tau_{n}>T \text { and } \rho_{n}>T\right\}}\right) & =\mathbb{E}\left(\sup _{0 \leq t \leq T}\left|e_{\Delta}(t)\right|^{p} 1_{\left\{v_{n}>T\right\}}\right) \\
& \leq \mathbb{E}\left(\sup _{0 \leq t \leq T}\left|x_{\Delta}\left(t \wedge v_{n}\right)-x\left(t \wedge v_{n}\right)\right|^{p}\right) .
\end{aligned}
$$

So by Lemma 4.5

$$
\mathbb{E}\left(\sup _{0 \leq t \leq T}\left|e_{\Delta}(t)\right|^{p} 1_{\left\{\tau_{n}>T \text { and } \rho_{n}>T\right\}}\right) \leq\left(C_{1}(n, p, T)+C_{2}(n, p, T)\right) \Delta^{p(1 / 4 \wedge \gamma)} e^{C_{3}(n, p, T)} .
$$

Also,

$$
\mathbb{P}\left(\tau_{n} \leq T \text { or } \rho_{n} \leq T\right) \leq \mathbb{P}\left(\tau_{n} \leq T\right)+\mathbb{P}\left(\rho_{n} \leq T\right) .
$$

Substituting the inequalities (48), (49) and (50) into (47), we obtain

$$
\begin{aligned}
\mathbb{E}\left(\sup _{0 \leq t \leq T}\left|e_{\Delta}(t)\right|^{p}\right) & \leq \frac{2^{p}\left(C_{1} \vee C_{4}\right) q \varrho}{p}+\left(C_{1}(n, p, T)+C_{2}(n, p, T)\right) \Delta^{p(1 / 4 \wedge \gamma)} e^{C_{3}(n, p, T)} \\
& +\frac{p-q}{p \varrho^{q /(p-q)}} \mathbb{P}\left(\tau_{n} \leq T\right)+\frac{p-q}{p \varrho^{q /(p-q)}} \mathbb{P}\left(\rho_{n} \leq T\right) .
\end{aligned}
$$

For any given $\varepsilon \in(0,1)$, we may choose $\varrho$ such that

$$
\frac{2^{p}\left(C_{1} \vee C_{4}\right) q \varrho}{p} \leq \frac{\varepsilon}{4}
$$

By Theorem 2.3 and Lemma 4.4 , for any given $\varepsilon \in(0,1)$, there exists $n_{o}$ such that for $n \geq n_{o}$ we may choose $\varrho$ to have

$$
\frac{p-q}{p \varrho^{q /(p-q)}} \mathbb{P}\left(\tau_{n} \leq T\right) \leq \frac{\varepsilon}{4}
$$

and choose $n(\varepsilon) \leq n_{o}$ such that for $\Delta \in\left(0, \Delta_{1}\right]$

$$
\frac{p-q}{p \varrho^{q /(p-q)}} \mathbb{P}\left(\rho_{n} \leq T\right) \leq \frac{\varepsilon}{4} .
$$

Lastly, we may select $\Delta \in\left(0, \Delta_{1}\right]$ sufficiently small for $\varepsilon \in(0,1)$ such that

$$
\left(C_{1}(n, p, T)+C_{2}(n, p, T)\right) \Delta^{p(1 / 4 \wedge \gamma)} e^{C_{3}(n, p, T)} \leq \frac{\varepsilon}{4} .
$$

Combining (51), (52), (53) and (54), we get

$$
\mathbb{E}\left(\sup _{0 \leq t \leq T}\left|x_{\Delta}(t)-x(t)\right|^{p}\right) \leq \varepsilon
$$

as the required assertion. 


\section{Applications to finance}

It is well known that the strong convergence of numerical solutions guarantees convergence in Monte Carlo simulations relevant for valuing many path dependent financial quantities (see e.g., [9]). In this section we apply the strong convergence result obtained in Theorem 4.6 to value some financial instruments such as a bond and a barrier option.

\subsection{Bond valuation}

If the short-term interest rate dynamics is described by SDDE (4), then the payoff of a bond at the end of time $T$ is given by

$$
B(T)=\mathbb{E}\left[\exp \left(-\int_{0}^{T} x(t)\right)\right] .
$$

Using the step function $\bar{x}(t)$ defined in $(29)$, the approximate payoff based on the truncated EM method becomes

$$
\bar{B}_{\Delta}(T)=\mathbb{E}\left[\exp \left(-\int_{0}^{T} \bar{x}_{\Delta}(t)\right)\right] .
$$

It then follows from Theorem 4.6 that

$$
\lim _{\Delta \rightarrow 0}\left|B(T)-\bar{B}_{\Delta}(T)\right|=0
$$

\subsection{Barrier option valuation}

Consider a barrier option with a European payoff $P$. Let the asset price be the exact solution $x(T)$ to SDDE (4) at expiry date $T, B$ a fixed barrier and $K$ a strike price. The payoff at expiry date is

$$
\left.P(T)=\mathbb{E}\left[(x(T)-K)^{+} 1_{\sup _{0 \leq t \leq T}} x(t)<B\right)\right] .
$$

The approximate payoff using the truncated EM scheme defined in $(29)$ becomes

$$
\left.P_{\Delta}(T)=\mathbb{E}\left[\left(\bar{x}_{\Delta}(T)-K\right)^{+} 1_{\sup _{0 \leq t \leq T}} \bar{x}_{\Delta}(t)<B\right)\right] .
$$

So from Theorem 4.6, we have

$$
\lim _{\Delta \rightarrow 0}\left|P(T)-\bar{P}_{\Delta}(T)\right|=0 .
$$

You may consult [9, 11] for detailed coverage of the proofs and further examples.

\section{$6 \quad$ Numerical Experiments}

To illustrate efficiency of our proposed truncated EM scheme for SDDE (4), we will perform two numerical examples with different Ait-Sahalia-type models. In the first numerical example, we will implement Ait-Sahalia-type model with $\alpha_{-1} x(t)^{-1}$ term in the drift and delayed volatility function. In the second numerical example, we will implement and perform comparative assessment of delayed Ait-Sahalia-type model without $\alpha_{-1} x(t)^{-1}$ term in the drift using both truncated EM (TEM) and backward EM (BEM) schemes. This becomes necessary because it is unknown if the backward EM scheme could cope with $\alpha_{-1} x(t)^{-1}$ term at the origin. We would like to point out that we consider 
this case and use BEM scheme in our numerical study because the main available literature for one half strong order approximation of Ait-Sahalia-type model focuses on the BEM method (see e.g [25]). There is so far no relevant literature devoted to strong convergent approximation of Ait-Sahalia-type model with $\alpha_{-1} x(t)^{-1}$ term and delayed volatility function.

\subsection{Numerical example I}

In this numerical illustration, we consider the following delayed Ait-Sahalia-type model

$$
d x(t)=\left(0.1 x(t)^{-1}-0.3+x(t)-0.5 x(t)^{3}\right) d t+V(x(t-1)) x(t)^{3 / 2} d B(t),
$$

with initial data $\xi(0)=0.2$ and $V(\cdot)$ is defined by

$$
V(y)= \begin{cases}\frac{1}{2} \frac{\left(1+\left(e^{y}-e^{-y}\right)\right)}{\left(e^{y}+e^{-y}\right)}, & \text { if } y \geq 0 \\ \frac{1}{4}, & \text { Otherwise }\end{cases}
$$

Note (56) is a special type of sigmoid function. Naturally, sigmoid functions like (56) are bounded, realvalued functions and hence fulfill Assumption 2.1. Moreover, parameterising sigmoid-based functions in financial models on past data are observed to capture volatility skews and smiles (see, e.g., [26]). Do also note the drift and diffusion coefficient terms of (55) satisfy

$$
\sup _{1 / u \leq x \leq u}(|f(x)| \vee g(x)) \leq 1.9 u^{3}, \quad u \geq 1
$$

This means we can have $\mu(u)=1.9 u^{3}$ with inverse $\mu^{-1}(u)=(u / 1.9)^{1 / 3}$. If we define $\Delta=10^{-3}$ and $h(\Delta)=\Delta^{-2 / 3}$, then $\mu^{-1}(h(\Delta))=\left(\Delta^{-2 / 3} / 1.9\right)^{1 / 3}$ and $1 / \mu^{-1}(h(\Delta))=\left(\Delta^{-2 / 3} / 1.9\right)^{-1 / 3}$. Displayed in Figure 1.0 is a Monte Carlo simulated sample path of $x(t)$ with step size $10^{-2}$ using the TEM scheme.

\subsection{Numerical example II}

In this subsection, we will assess the performance of TEM scheme with BEM scheme. We already noted there exists no relevant literature on strong convergent approximation of SDDE (57). Hence, we have to fall on the BEM method which has 0.5 strong order approximation of Ait-Sahalia-type model without the delayed volatility function. Consider the following delayed Ait-Sahalia-type model

$$
d x(t)=\left(0.2+0.3 x(t)-0.5 x(t)^{2}\right) d t+V(x(t-1)) x(t)^{4 / 3} d B(t),
$$

with initial data $\xi(0)=0.2$ and the same volatility function $V(\cdot)$ in $(56)$. Clearly, we have $\mu(u)=u^{2}$ with inverse $\mu^{-1}(u)=u^{1 / 2}$. Using TEM and BEM schemes with step size $10^{-3}$, we obtain Monte Carlo simulated sample paths of $x(t)$ in Figure 2.0. We notice that both simulated sample paths are almost the same. Figure 3.0 depicts log-log plot of the strong errors between TEM and BEM schemes using step sizes $10^{-3}, 10^{-4}, 10^{-5}$ and $10^{-6}$. For the purpose of comparison, we also plotted the reference line with slop 1.0. We can see the strong error between TEM and BEM schemes has order 1.0 though this has not been proved theoretically. . 


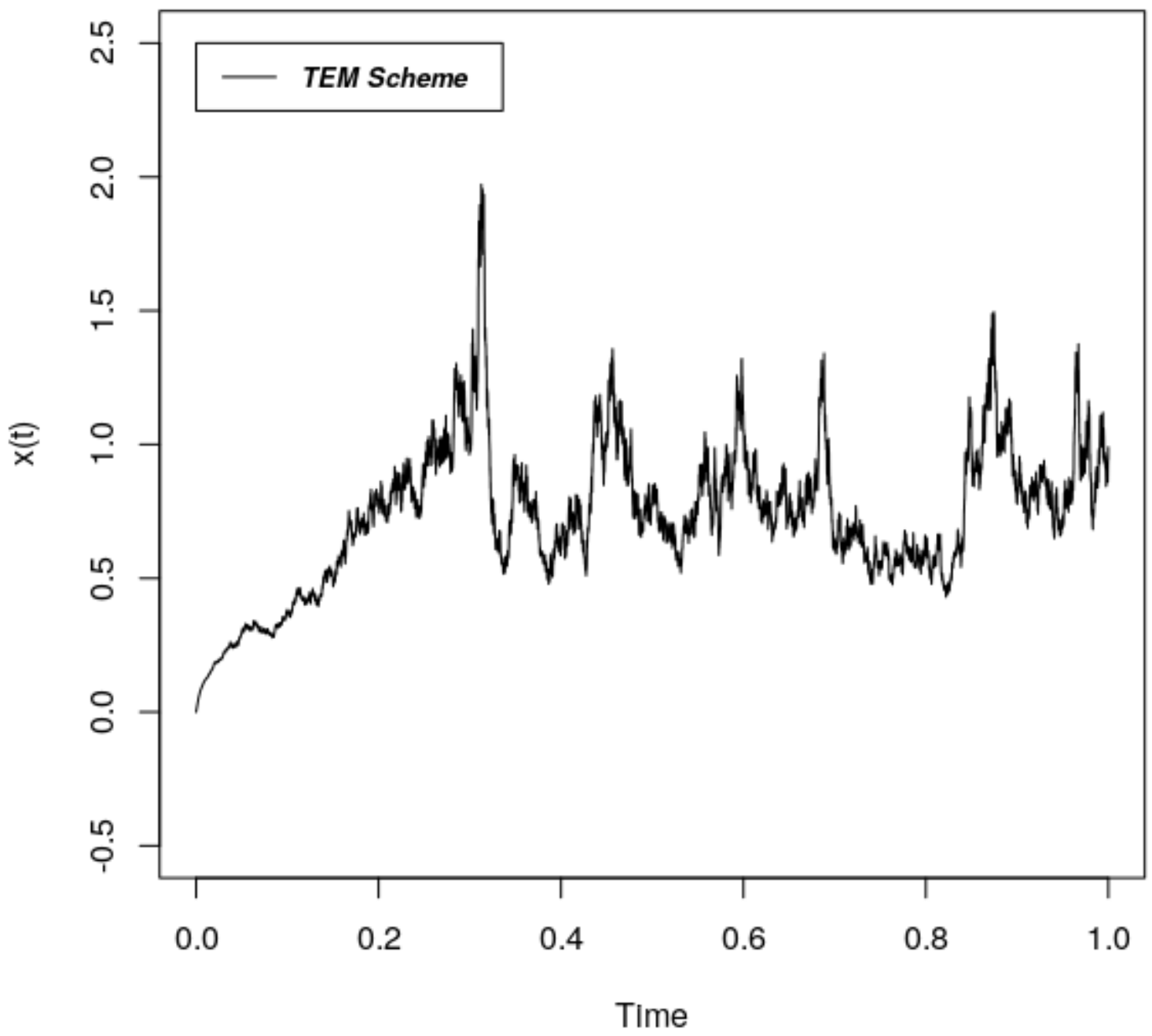

Figure 1: Monte Carlo simulated sample path of $x(t)$ by TEM scheme. 


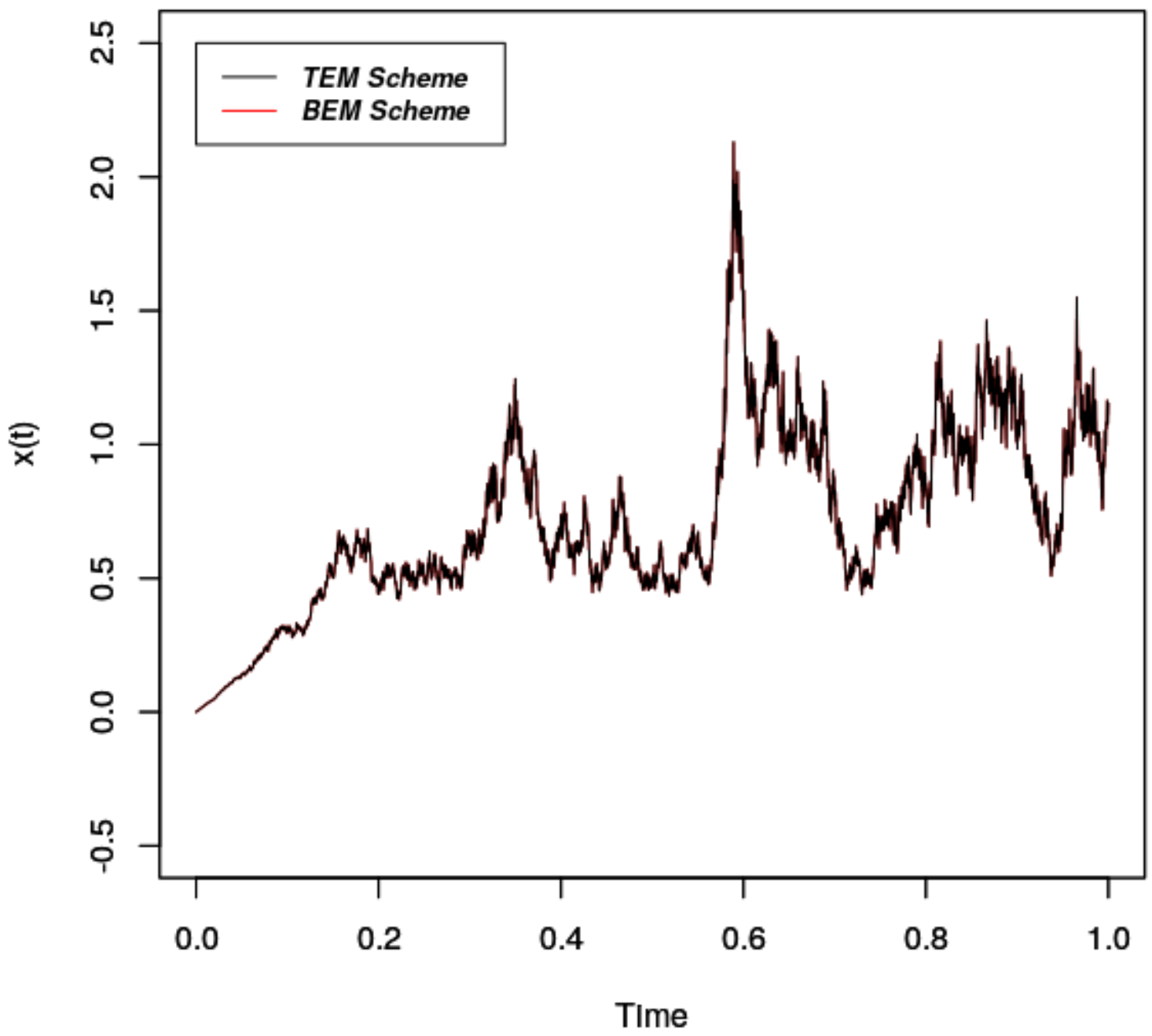

Figure 2: Monte Carlo simulated sample paths of $x(t)$ by TEM and BEM schemes. 


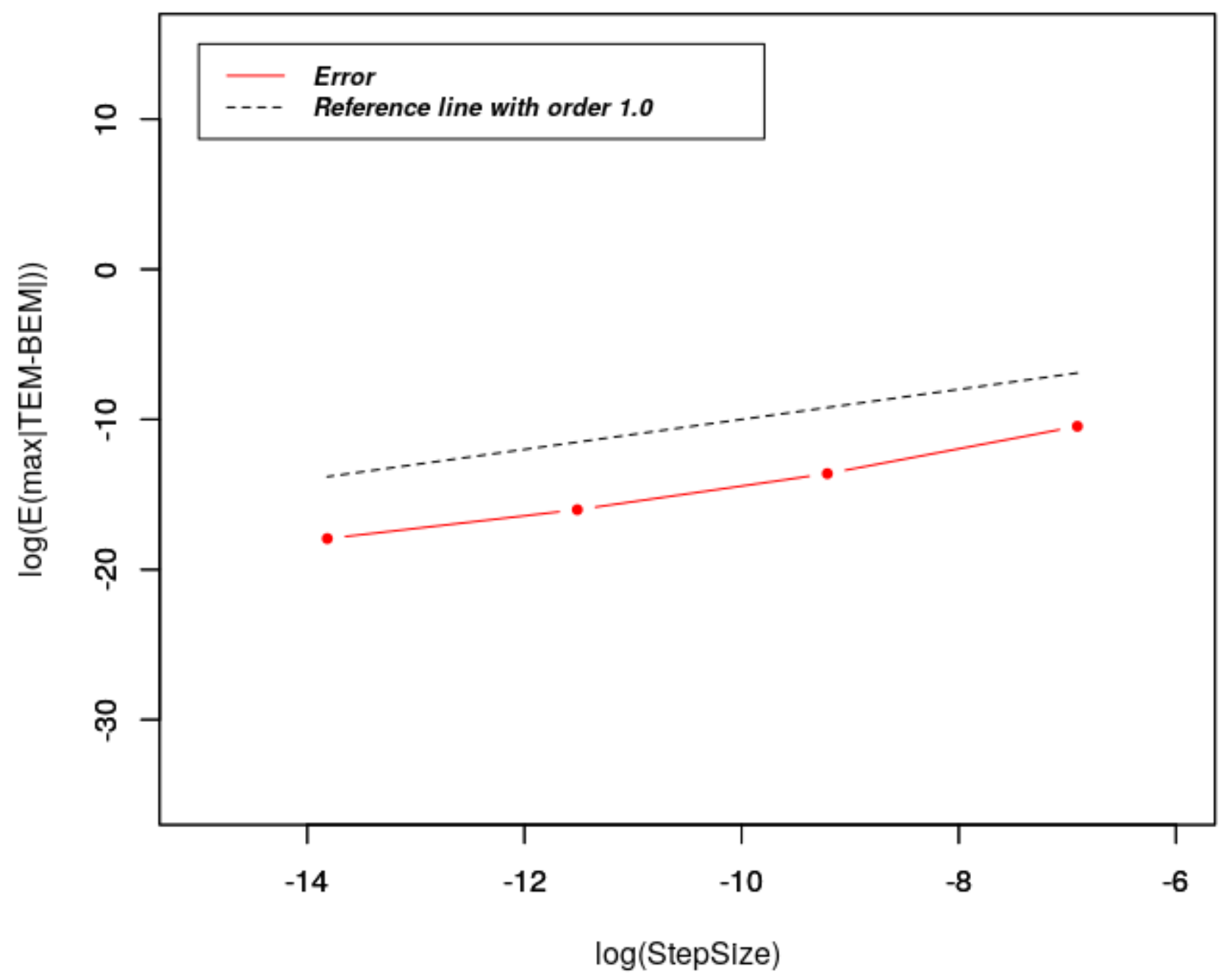

Figure 3: The strong errors between TEM and BEM schemes. 


\section{Acknowledgements}

The authors would like to thank the editor and reviewers for their helpful comments and suggestions. The first Author would like to thank the University of Strathclyde for the financial support. The second author would like to thank the Royal Society (WM160014, Royal Society Wolfson Research Merit Award), the Royal Society and the Newton Fund (NA160317, Royal Society-Newton Advanced Fellowship).

\section{References}

[1] Black, F. and Scholes, M., 1973. The pricing of options and corporate liabilities. Journal of political economy, 81(3), pp.637-654.

[2] Merton, R.C., 1973. Theory of rational option pricing. Theory of Valuation, pp.229-288.

[3] Vasicek, O., 1977. An equilibrium characterization of the term structure. Journal of financial economics, 5(2), pp.177-188.

[4] Dothan, L.U., 1978. On the term structure of interest rates. Journal of Financial Economics, 6(1), pp.59-69.

[5] Brennan, M.J. and Schwartz, E.S., 1980. Analyzing convertible bonds. Journal of Financial and Quantitative analysis, 15(4), pp.907-929.

[6] Cox, J.C., Ingersoll Jr, J.E. and Ross, S.A., 1980. An analysis of variable rate loan contracts. The Journal of Finance, 35(2), pp.389-403.

[7] Cox, J.C., Ingersoll Jr, J.E. and Ross, S.A., 2005. A theory of the term structure of interest rates. In Theory of Valuation (pp. 129-164).

[8] Lewis, A.L., 2009. Option Valuation Under Stochastic Volatility Ii. Finance Press, Newport Beach, CA.

[9] Higham, D.J. and Mao, X., 2005. Convergence of Monte Carlo simulations involving the meanreverting square root process. Journal of Computational Finance, 8(3), pp.35-61.

[10] Wu, F., Mao, X. and Chen, K., 2008. A highly sensitive mean-reverting process in finance and the Euler-Maruyama approximations. Journal of Mathematical Analysis and Applications, 348(1), pp. $540-554$.

[11] Mao, X., 2007. Stochastic differential equations and applications. 2nd ed. Chichester: Horwood Publishing Limited.

[12] Ait-Sahalia, Y., 1996. Testing continuous-time models of the spot interest rate. The review of financial studies, 9(2), pp.385-426.

[13] Cheng, S.R., 2009. Highly nonlinear model in finance and convergence of Monte Carlo simulations. Journal of Mathematical Analysis and Applications, 353(2), pp.531-543.

[14] Szpruch, L., Mao, X., Higham, D.J. and Pan, J., 2011. Numerical simulation of a strongly nonlinear Ait-Sahalia-type interest rate model. BIT Numerical Mathematics, 51(2), pp.405-425. 
[15] Dung, N.T., 2016. Tail probabilities of solutions to a generalized Ait-Sahalia interest rate model. Statistics and Probability Letters, 112, pp.98-104.

[16] Hutzenthaler, M., Jentzen, A. and Kloeden, P.E., 2010. Strong and weak divergence in finite time of Euler's method for stochastic differential equations with non-globally Lipschitz continuous coefficients. Proceedings of the Royal Society A: Mathematical, Physical and Engineering Sciences, $467(2130)$.

[17] Andersen, T.G., Bollerslev, T., Diebold, F.X. and Labys, P., 2001. The distribution of realized exchange rate volatility. Journal of the American statistical association, 96(453), pp.42-55.

[18] Hull, J. and White, A., 1987. The pricing of options on assets with stochastic volatilities. The journal of finance, 42(2), pp.281-300.

[19] Kind, P., Liptser, R.S. and Runggaldier, W.J., 1991. Diffusion approximation in past dependent models and applications to option pricing. The Annals of Applied Probability, 1(3), pp.379-405.

[20] Mao, X. and Sabanis, S., 2013. Delay geometric Brownian motion in financial option valuation. Stochastics An International Journal of Probability and Stochastic Processes, 85(2), pp.295-320.

[21] Mao, X., 2015. The truncated Euler-Maruyama method for stochastic differential equations. Journal of Computational and Applied Mathematics, 290, pp.370-384.

[22] Guo, Q., Mao, X. and Yue, R., 2018. The truncated Euler-Maruyama method for stochastic differential delay equations. Numerical Algorithms, 78(2), pp.599-624.

[23] Mao, X. and Sabanis, S., 2003. Numerical solutions of stochastic differential delay equations under local Lipschitz condition. Journal of computational and applied mathematics, 151(1), pp.215-227.

[24] Mao, X., 2016. Convergence rates of the truncated Euler-Maruyama method for stochastic differential equations. Journal of Computational and Applied Mathematics, 296, pp.362-375.

[25] Higham, D.J., Mao, X. and Stuart, A.M., 2002. Strong convergence of Euler-type methods for nonlinear stochastic differential equations. SIAM Journal on Numerical Analysis, 40(3), pp.10411063.

[26] Itkin, A., 2015. To sigmoid-based functional description of the volatility smile. The North American Journal of Economics and Finance, 31, pp.264-291. 